AL.2.2003-215

c. 2

\title{
MACROFUNGI \\ OF \\ LA BUTTE CREEK, \\ FIDLER-GREYWILLOW AND \\ COLIN-CORNWALL \\ WILDLAND PROVINCIAL PARKS
}

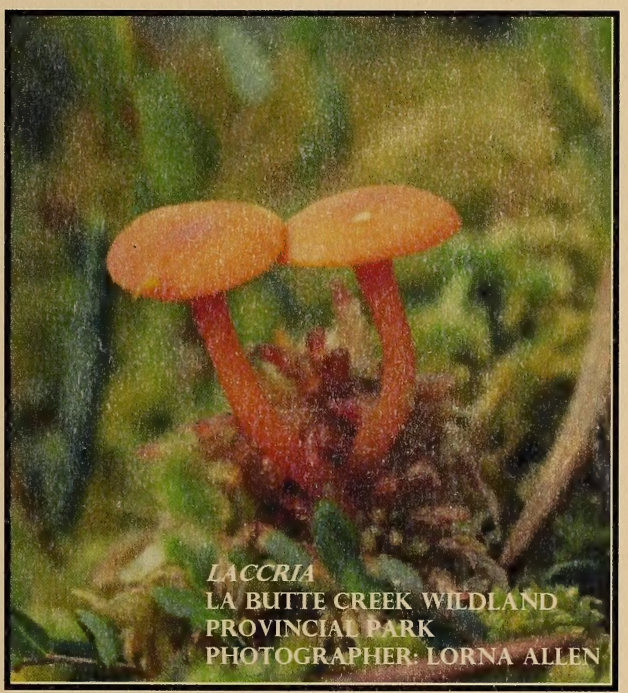

PREPARED FOR:

ALBERTA COMMUNITY DEVELOPMENT PARKS AND PROTECTED AREAS DIVISION

PREPARED BY:

WILLIAM RICHARDS AND DIANE MURRAY

JANUARY 2003 



\section{Executive Summary}

During 2001 and 2002 a project to collect and identify the macrofungi of La Butte Creek, Fidler-Greywillow and Colin-Cornwall Lakes Wildland Provincial Parks, in northeastern Alberta was undertaken. This project was part of a greater biophysical investigation carried out by Parks and Protected Areas' staff, and volunteers from a variety of scientific backgrounds.

The fungi found belong to three major groups; the Ascomycota (cup fungi), Basidiomycota (mushrooms, rusts, smuts, etc.) and Myxomycota (slime molds).

One hundred of the 156 species documented through this study have been previously recorded as occurring in Alberta. Valid Alberta records have yet to be found for the remaining 56 species. Also two species discovered (Gloeophyllum abietinum and Myriosclerotinia caricicampullaceae) are reportedly rare in North America.

\section{This report may be cited as:}

Richards, W. M. and D. M. Murray. 2002. Macrofungi of La Butte Creek, Fidler-Greywillow and Colin-Cornwall Lakes Wildland Provincial Parks, Alberta. Prepared for Alberta Community Development, Parks and Protected Areas Division, Edmonton, Alberta. 
Introduction.

Methods

Results.

Discussion

Summary.....

Recommendations.

Literature Cited.

General References. 8

List of Figures ..iv

Appendix 1.

Taxonomic listing of macrofungi from La Butte Creek, Fidler-Greywillow and

Colin-Cornwall Lakes Wildland Provincial Parks with note of previous Alberta records

Appendix 2.

Annotated species list for La Butte Creek Wildland Provincial Park.

Appendix 3.

Annotated species list for Fidler-Greywillow Wildland Provincial Park

Appendix 4.

Annotated species list for Colin-Cornwall Lakes Wildland Provincial Park

Appendix 5.

Partial list of literature accounts of fungi occurrences in Alberta .24

Appendix 6.

Photographs of macrofungi from La Butte, Fidler-Greywillow and Colin-Cornwall Lakes Wildland Provincial Parks. 


\section{List of Figures}

Map 1. Location of La Butte Creek, Fidler-Greywillow and Colin-Cornwall Lakes Wildland

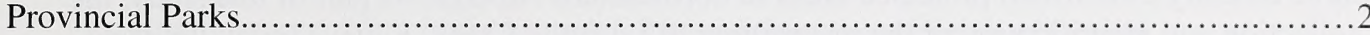




\section{Introduction}

During July 2001 and July 2002 fieldwork was undertaken by staff of Parks and Protected Areas Division of Alberta Community Development, and volunteers from a variety of scientific backgrounds. The purpose of the fieldwork was to collect baseline biophysical information in three recently established protected areas in northeastern Alberta. As part of this study, the collection and identification of the macrofungi of La Butte Creek, Fidler-Greywillow and Colin-Cornwall Lakes Wildland Provincial Parks (see Map 1) was conducted. This paper represents a snapshot of the mycological flora of these sites, and by no means comprises a definitive listing of fungi for these areas.

These parks are primarily within the Kazan Upland Sub-region of the Canadian Shield Natural Region in northeastern Alberta. La Butte Creek Wildland Provincial Park contains diverse areas of wetland complexes and Precambrian shield outcrops. La Butte Creek itself is a large stream draining from the Kazan Upland. A small portion of the park is bordering on the Slave River in the Peace River Lowland Sub-region.

Fidler-Greywillow Wildland Provincial Park contains one of the most diverse sections of the north shore of Lake Athabasca in Alberta. It has extensive sandy beaches, beach ridges and bedrock outcrops. This area contains the best exposure of Athabasca Sandstone outcrops in the region and has plant species not found south of Lake Athabasca. The park also incorporates a number of islands, including Bustard Island, the largest island in the province.

Colin-Cornwall Lakes Wildland Provincial Park contains diverse areas of wetland complexes and Precambrian shield outcrops. The park is primarily within the Kazan Upland Sub-region.

\section{Methods}

Observations and collections were limited to the macrofungi. Macrofungi form "fruiting bodies" that are visible to the naked eye and are generally greater than $1 \mathrm{~cm}$ in diameter (Redhead 1997). These fruiting bodies are the mushrooms, toadstools and puff-balls that are commonly perceived by the public as "mushrooms". Both terrestrial and arboreal fungi were collected. Fungi were searched for at the microhabitat level (Redhead and Berch 1997), where searches included such habitats as: woody plants (living trees and shrubs); shed bark; leaf and needle litter; course woody debris (dead trees, standing or fallen); beaches and shorelines of lakes, wetlands and creeks; bogs; soil; burn sites and other areas of organic accumulation.

Flying squirrel and other rodents include truffle and secotioid fungi in their diet (Zabel and Walters 1997 \& Currah et al 2002). For this reason subterranean searches for truffle and false truffles were undertaken at all locations where evidence of rodent digging was noted. This search required scraping and sifting through a large quantity of duff using a three prong garden fork. Searches were restricted to the humus layer and covered an area approximately $40 \mathrm{~cm}$ square, which centered on the rodent's original excavation.

As specimens were collected they were placed in numbered and labeled waxed paper bags. Notes on habitat, species name or family (if known); and specimen and spore colour were included. Where spore colour was not readily discernible but required for identification, an 
attempt to acquire a spore print was made by placing the specimen on white paper before depositing it in the waxed bag.

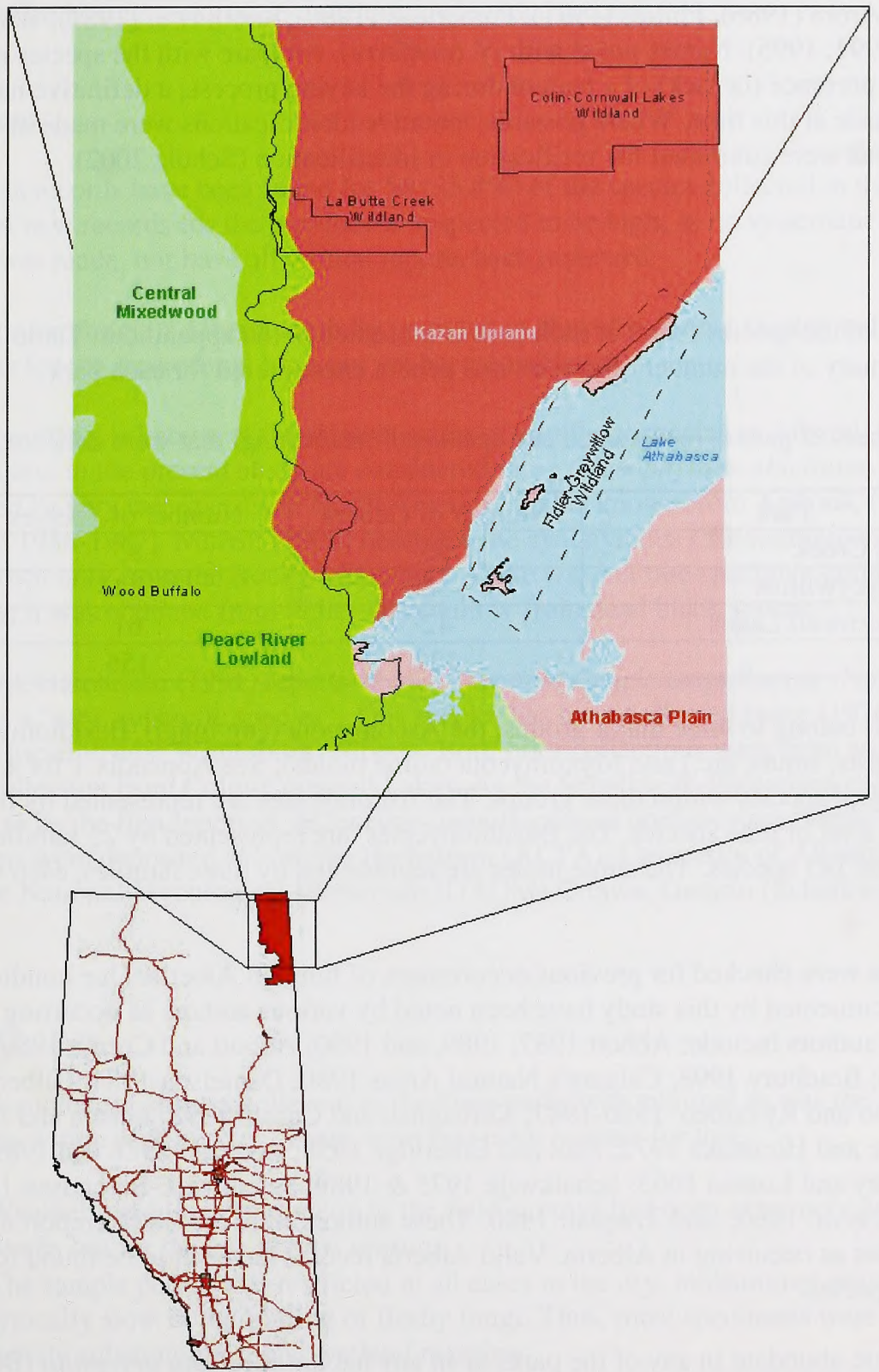

Map 1. Locations of Fidler-Grewwillow, La Butte Creek and Collin-Cornwall Lakes Wildlands 
Scientific names are based on current nomenclature. Different references were used to identify the different groups of fungi as follows: Brandrud et al. $(1990,1992,1994,1998)$ was used for Cortinarius; Overholts (1967) and Gilbertson and Ryvarden (1986-1987) for the polypore groups; Martin and Alexopolos (1969) for the Myxomycetes; Smith et al (1979) and Kauffman (1918) for the Agarics; and Moser (1983) for Polyporales, Boletales, Agaricales, and Russulales. In addition several important works were used as general identification guides, these include: Arora (1986), Philips (1991), Pomerleau (1980), Smith et al (1981), Walters et al (1981, 1986, 1991, 1995). Names noted with $c f$. (conferre), compare with the species noted but because of the presence (or lack) of a feature during the keying process, a definitive naming could not be made at this time. Where possible, tentative identifications were made in the field, but all specimens were submitted for verification or identification (Schulz 2002).

\section{Results}

A complete list of the species found at each park is presented in the appendices. Table 1 contains a summary of the number of species and genera encountered for each park.

Table 1. Numbers of genera represented and species of macrofungi that were collected in three parks.

\begin{tabular}{|l|c|c|}
\hline \multicolumn{1}{|c|}{ Park } & Number of Genera & Number of Species \\
\hline La Butte Creek & 55 & 85 \\
\hline Fidler-Greywillow & 32 & 64 \\
\hline Colin-Cornwall Lakes & 42 & 61 \\
\hline Total & 129 & 156 \\
\hline
\end{tabular}

The fungi found belong to three major groups; the Ascomycota (cup fungi), Basidiomycota (mushrooms, rusts, smuts, etc.) and Myxomycota (slime molds). See Appendix 1 for a complete listing of species within these groups. The Ascomycetes are represented by five families with a total of nine species. The Basidiomycetes are represented by 25 families and contain a total of 143 species. The slime moles are represented by three families, each with one species.

Various sources were checked for previous occurrences of fungi in Alberta. One hundred of the 156 species documented by this study have been noted by various authors as occurring in Alberta. These authors include: Abbott 1987, 1989, and 1990; Abbott and Currah 1989; Baranyay 1968; Bradbury 1998; Calgary's Natural Areas 1980; Danielson 1984; Gilbertson 1981; Gilbertson and Ryvarden 1986-1987; Kernaghan and Currah 1998; Larsen and Denison 1978; Lawrence and Hiratsuka 1972; Paul and Etheridge 1958; Redhead 1988 and 1989; Robinson-Jeffrey and Loman 1963; Schalkwijk 1975 \& 1989; Schalkwijk-Barendsen 1991 \& 1991a; Thomas et al. 1960; and Traquair 1980. These authors were the first to report a particular species as occurring in Alberta. Valid Alberta records have yet to be found for the remaining 56 species.

Few species were abundant in any of the parks or in any habitat. Collybia dryophila (Bull.:Fr) Kum. within the Family Tricholomataceae was represented at each park, occurring a total of 15 times, and were the most common fungi at La Butte Creek with 10 occurrences. There was a total of 28 species represented within the Tricholomataceae and 31 within the Family 
Polyporaceae. The Family Cortinariaceae had 36 species recorded. There were 90 species encountered only once during this study.

No truffles (in the Family Tuberaceae) or false truffles (in the Family Rhizopogonaceae) were discovered in the process of searching rodent dig sites. However, at several locations small gelatinous globules resembling false truffles were discovered. These globules were later determined to be egg sacks of an undetermined spider species.

\section{Discussion}

No Alberta records have been found for $56(35.8 \%)$ of the species collected in this study. This number of new records for the province is suspected to be high, as no systematic search of herbaria was made, nor have all written reports been reviewed.

Only the microfungi of Alberta are fully recorded in checklist format (Sigler and Flis 1998). Checklists for the macrofungi have not yet been completed.

Virtually nothing is known of the relative status of any fungi species in Alberta, however two species found in the present study are considered rare. Gloeophyllum abietinum (Fr.) Karst., is considered "a very rare species in North America" and is known from Arizona, (Gilbertson and Ryvarden 1986-1987). Murrill (1908) describes the synonymous G. abietinellum Murr. as being known only from the Rocky Mountains. There was but one specimen collected during this study; it was obtained from Fidler-Greywillow from dead black spruce.

Schalkwijk-Barendsen (1991) reports Myriosclerotinia caricic-ampullaceae (Nyberg.) N.F. Buchw as a "rare, subarctic species". This species has been collected twice (1976 and 1977) at the same location near Winterburn, Alberta. Both prior collections were from sedge as was the present collection from Colin-Cornwall Lakes. As the Schalkwijk-Barendsen collections were believed to be the first report of M. caricic-ampullaceae in northwestern North America, these collections were deposited at both the Herbarium (ALTA), University of Alberta, Edmonton, and at the National Mycological Herbarium (DAOM) Ottawa, Ontario (Schalkwijk-Barendsen 1991).

\section{Summary}

The total number of species collected in the three parks was minimal as was the number of specimens within each species. There were two main reasons for this:

- Weather conditions leading up to the field surveys had been extremely dry, not just for weeks but for the last several seasons.

- The sample period was restricted in all cases to the dry, midsummer season, which is typically slow in the fruiting of fleshy fungi. Thus, most specimens were obtained from woody substrates or along wetland margins. 
The sample period for each location was short and not repeated. According to Redhead and Berch (1997), "all possible sites and microhabitats within these sites must be examined repeatedly as seasons progress". Repeated visits to these remote sites were not, however, possible.

Of the 156 species of fungi documented, $35.8 \%$ (56 species) were not previously reported for Alberta. The number of apparently new species is high, and probably related to the following factors:

- No systemic review of herbaria was done for fungi;

- Fungi are not often included in biophysical studies, so are under-reported.

- A through review of the literature pertaining to fungi in Alberta was not completed.

In Alberta, the macrofungi species have not been ranked for "rarity", however:

- Two species collected during this study are considered rare: Gloeophyllum abietinum, (Gilbertson and Ryvarden 1986-1987) and Myriosclerotinia caricic-ampullaceae, (Schalkwijk-Barendsen 1991).

- Other species may also be rare, but that is impossible to substantiate at this time due to lack of information.

Fungi are one of the most important groups of organisms on the planet. They are responsible for most of the recycling of organic material back to the soil in a form that can be reused by other organisms. Fungi are vitally important for the growth of trees and most other plants, through mycorrhizal association. Currah and Van Dyk (1986) have, in one study, documented mycorrhizal association with 179 species of perennial plants native to Alberta.

\section{Recommendations}

There is a lack of knowledge of the macrofungi in Alberta. More time and research need to be devoted to understanding this group of organisms in the province.

These needs can be achieved by the following two steps:

1. Compile all known records from the existing literature for macrofungi within Alberta (Appendix 5 contains a partial list).

2. Compile all herbarium records for collections of macrofungi in Alberta.

Once these have been accomplished, provincial-wide surveys should be instituted. At a minimum, fungi investigations should be a part of all future biophysical assessments within the province. This will provide a starting point on which to base status assessments of the various species. 


\section{Literature Cited}

Abbott, S. P. 1987. The First Foray. The Stinkhorn, 1 (1): 12. Edmonton.

Abbott, S. P. 1989. Key to Boleti of Alberta. University of Alberta, Edmonton. 12pp. Mimeograph.

Abbott, S. P. 1990. Shaw Lake. The Stinkhorn, 4 (1): 38-41. Edmonton.

Abbott, S.P. and R.S. Currah. 1989. The larger cup fungi and other Ascomycetes of Alberta, an annotated checklist. Devonian Botanic Garden, University of Alberta, Edmonton. 96pp.

Baranyay, J.A. 1968.Fungi collected during forest diseases surveys in northern Alberta and the district of Mackenzie, Northwest Territories. For Branch Dept. Public no. 1238, 25p.

Bradbury, S.M., Danielson, R.M., and Visser, S. 1998. Ectomycorrhizas of regenerating stands of lodgepole pine (Pinus contorta). Can. J. Bot. 76: 218-227.

Brandrud, T. E. , Linström, H., Merlund, H., Melot, J., and Muskos, S. 1990, 1992. 1994, 1998. Cortinarius Flora Photographica. Vol. 1-4. Cortinarius HB, Matfors, Sweden (Cortinarius).

Calgary's Natural Areas. 1980, A Report Prepared by the Natural Areas Committee of the Calgary Field Naturalists Society, December 1980. Harold W. Pinel, Editor. (on line at http://weaselhead.org/learn/slimesmoldsfungi.htm; viewed <last date February 10, 2003>).

Currah, R.S., and Van Dyk, M. 1986. A survey of some perennial vascular plant species native to Alberta for the occurrence of mycorrhizal fungi. Can. Field-Nat. 100: 330-342.

Currah RS; Smreciu EA; Lehesvirta T; Niemi M; Larsen KW. 2000. Fungi in the winter diets of northern flying squirrels and red squirrels in the boreal mixedwood forest of northeastern Alberta. Canadian Journal of Botany-Revue Canadienne de Botanique. 78(12):1514-1520.

Danielson, R.M. 1984. Ectomycorrhizal associations in jackpine stands in northeastern Alberta, Can. J. Bot. 62:932-939.

Gilbertson, R.L. 1981. North American wood-rotting fungi that cause brown rots. Mycotaxon 12:372-416.

Gilbertson, R.L. and L. Ryvarden. 1986-1987. North American polypores. Fungifiora Vols. 12. Oslo, Norway. (Polypores).

Hiratsuka, Y. 1987. Forest tree diseases of the Prairie Provinces. Env. Can. North. For. Res. Cent. Inf. Rep. No. NOR-X-286

Kauffman, C. H. 1918. The Agaricaceae of Michigan. Wynkoop Hallenback Crawford Co., State Printers, Lansing, Michigan, USA (Agarics).

Kernaghan, G. and Currah, R. S. 1998. Ectomycorrhizal fungi at tree line in the Canadian Rockies. Mycotaxon 69: 39-80.

Lawrence J. J. and Hiratsuka. 1972. Forest fungi collection in Banff National Park, Alberta. Information Report NOR-X-22, Northern Forest Research Centre.

Larsen, H. J. and W. C. Denison. 1978. A checklist of operculate cup-fungi (Pezizales) of North America west of the Great Plains. Mycotaxon 7:68-90.

Martin, G. W. and Alexopolos, C. J. 1969. The Myxomycetes. University of Iowa Press, Iowa City, USA (Myxomycetes).

Moser, M. (1983). Keys to Agarics and Boleti (Polyporales, Boletales, Agaricales, Russulales) [English translation by Plant, S., ed. Kibby, G.].

Natural Resources Canada. 1996. Aspen trunk rot. Pages 52-53 in Common tree diseases of British Columbia. Canadian Forest Service, Pacific Forestry Centre, Victoria, BC. Edited by E.A. Allen, D.J. Morrison, and G. Wallis. 178 p. (on-line at 
http://www.pfc.cfs.nrcan.gc.ca/diseases/CTD/Group/Heart/heart14 e.html; viewed <last date February 26, 2003>).

Overholts, L. O. 1967. The Polyporaceae of the United States, Alaska, and Canada. The University of Michigan Press, Michigan, USA (Polypores).

Paul, G. and Etheridge, D.E. 1958. Decay of aspen (Populus tremuloides Michx.) and balsam poplar (P. balsamifera L.) in the Lesser Slave Lake region of Alberta. Alberta Dep. Land For., For. Serv. Div., Edmonton, Alberta, and Can. Dep. Agric., For. Biol. Div., V+Calgary, Alberta, Mimeograph.

Redhead, S.A. 1988. Notes on the genus Xeromphalina (Agaricales, Xerulaceae) in Canada: biogeography, nomenclature, taxonomy. Can. J. Bot. 66:479-507.

Redhead, S.A. 1989. A biogeographical overview of the Canadian mushroom flora. Can. J. Bot. 67:3003-62.

Redhead, S.A. 1997. Macrofungi of British Columbia: requirements for inventory. Res. Br., B.C. Min. For., and Wildl. Br. B.C. Min. Envirn., Lands and Parks. Victoria, B.C. Work Pap. 28/1997. 119 pgs.

Redhead, S.A., and S. Berch. 1997. Standardized Inventory Methodologies for Components of British Columbia's Biodiversity: MACROFUNGI (including the phyla Ascomycota and Basidiomycota). Version 1.1. Resources inventory committee approved standard. British Columbia.

Robinson-Jeffrey, R. C., and A. A. Loman. 1963. Fungi Isolated in Culture from Red Heartwood Stain and Advance decay of Lodgepole Pine in Alberta. Can. J. Bot. 41:1371-1375.

Schalkwijk, H.M.E. 1975. Mushrooms of the Edmonton Area - edible and poisonous. Printed and published in Edmonton 34pp.

Schalkwijk, H.M.E. 1989. Leni Schalkwijk's checklist of Alberta Fungi. The Stinkhorn, Edmonton 3 (1): 1-66.

Schalkwijk-Barendsen, H. M. E. 1991. Mushrooms of Western Canada. Lone Pine Publishing, Edmonton, Alberta, Canada.

Schalkwijk-Barendsen, H.M.E. 1991a. Checklist to accompany the book - Mushrooms of Western Canada, published by author.

Schulz, M. 2002. Personal Commutations. Mycologist, Department of Biological Science. University of Alberta. Edmonton Alberta.

Sigler, L. and A.L. Flis. 1998. University of Alberta microfungus collection and herbarium (UAMH) Devonian Botanic Garden, Edmonton, AB, Canada.

Smith, A. H., Smith, H. V., and Weber, N.S. 1979. How to Know the Gilled Mushrooms. Wm. C. Brown Company Publishers, Dubuque, Iowa, USA (Agarics).

Thomas, G.P., D. E. Etherridge and G. Paul. 1960. Fungi and decay in aspen and balsam poplar in the boreal forest region, Alberta. Can J. Bot. 38: 459-466.

Traquair, J.A. 1980. The first Mary Elliot Mycological foray. Alberta Naturalist. 10: 4-6.

Zabel, C. J. and J. R. Waters. 1997. Food preferences of captive northern flying squirrels from the Lassen National Forest in northeastern California. Northwest Science, 71:103-107.

Zeglen, S. 1997 . Tree wounding and partial-cut harvesting: a literature review for British Columbia (Pest management report ; no. 14) Ministry of Forests, Pacific Forestry Centre, Canadian Forest Service. 


\section{General References}

Arora, D. 1986. Mushrooms Demystified. Ten Speed Press, Berkley, California, USA (General). Philips, R. 1991. Mushrooms of North America. Little, Brown and Company, Toronto, Ontario, Canada (General).

Pomerleau, R. 1980. Flore des Champignons au Quebec. Les Editions la Presse, Ltee, Ottawa, Canada (General).

Smith, A. H., Smith, H. V., and Weber, N.S. 1981. How to Know the Non-gilled Mushrooms. Wm. C. Brown Company Publishers, Dubuque, Iowa, USA (General non-Agarics).

Walters, V. L., Breitenbach, J., and Kränzlin, F. 1981. 1986, 1991. 1995. Fungi of Switzerland. Vol. 1-4. Verlag Mykologia, Luzern, Switzerland (General). 


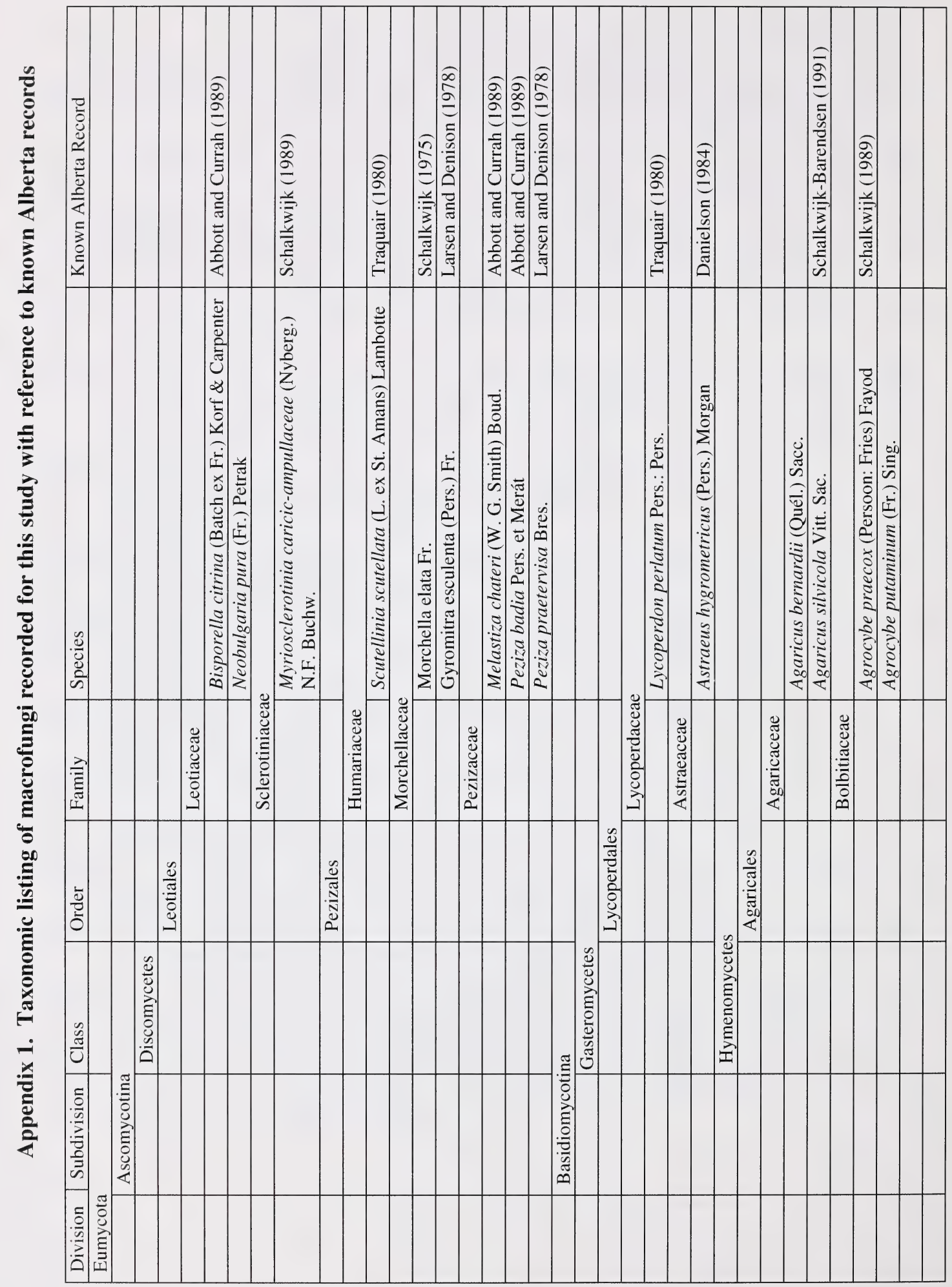




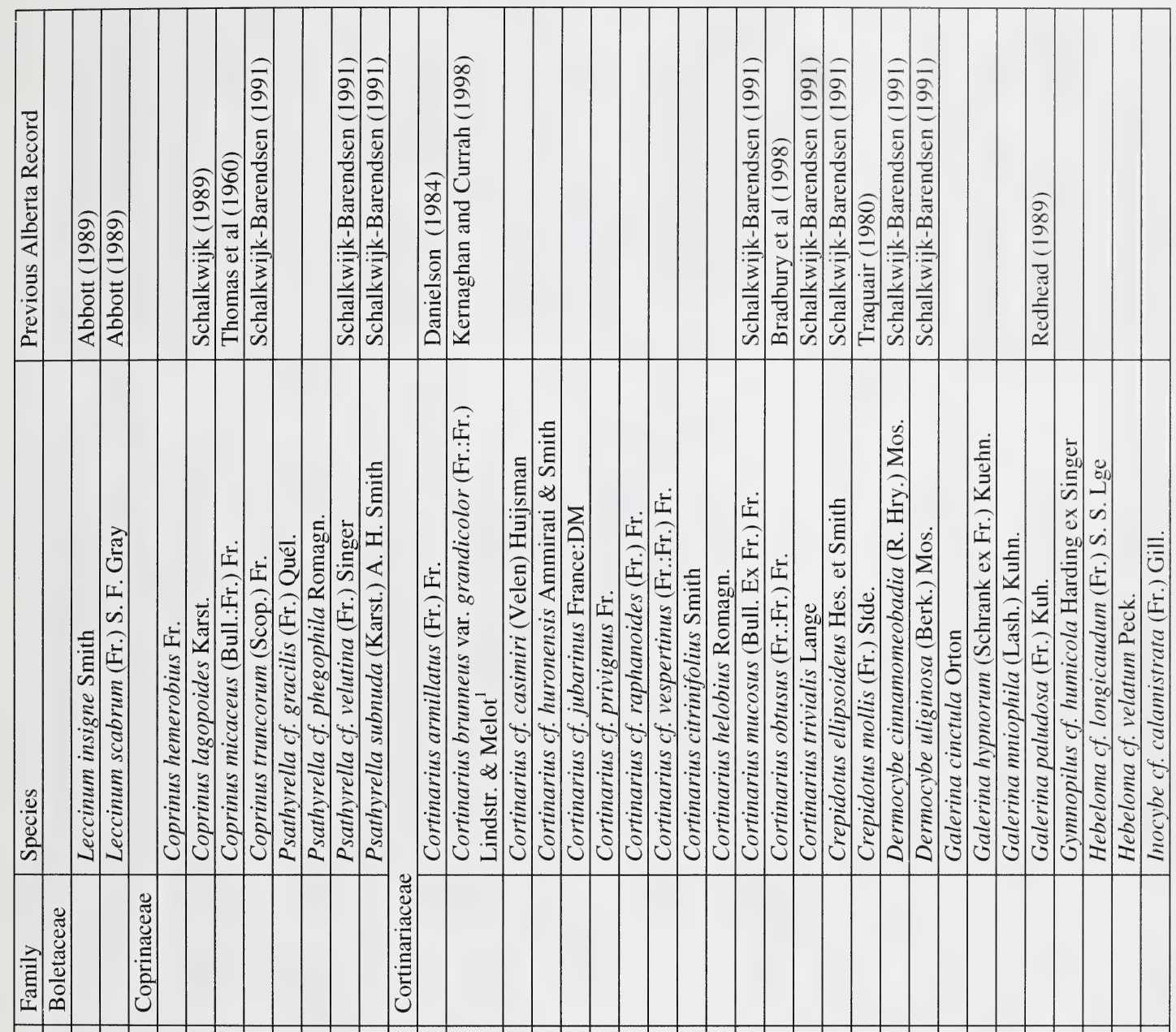

홍

ปี

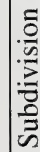

흘 

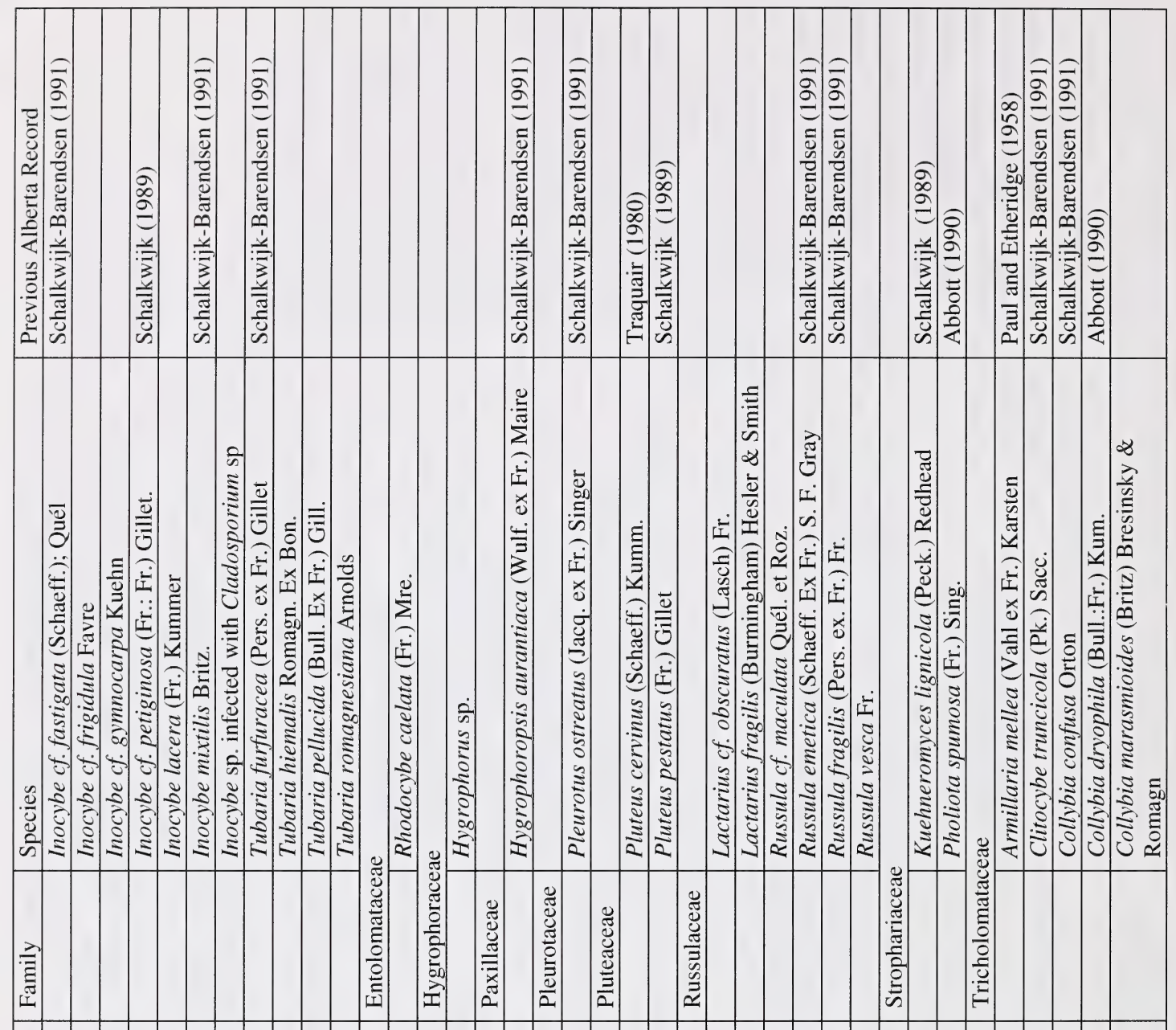

$\frac{5}{5}$

急

:

童 

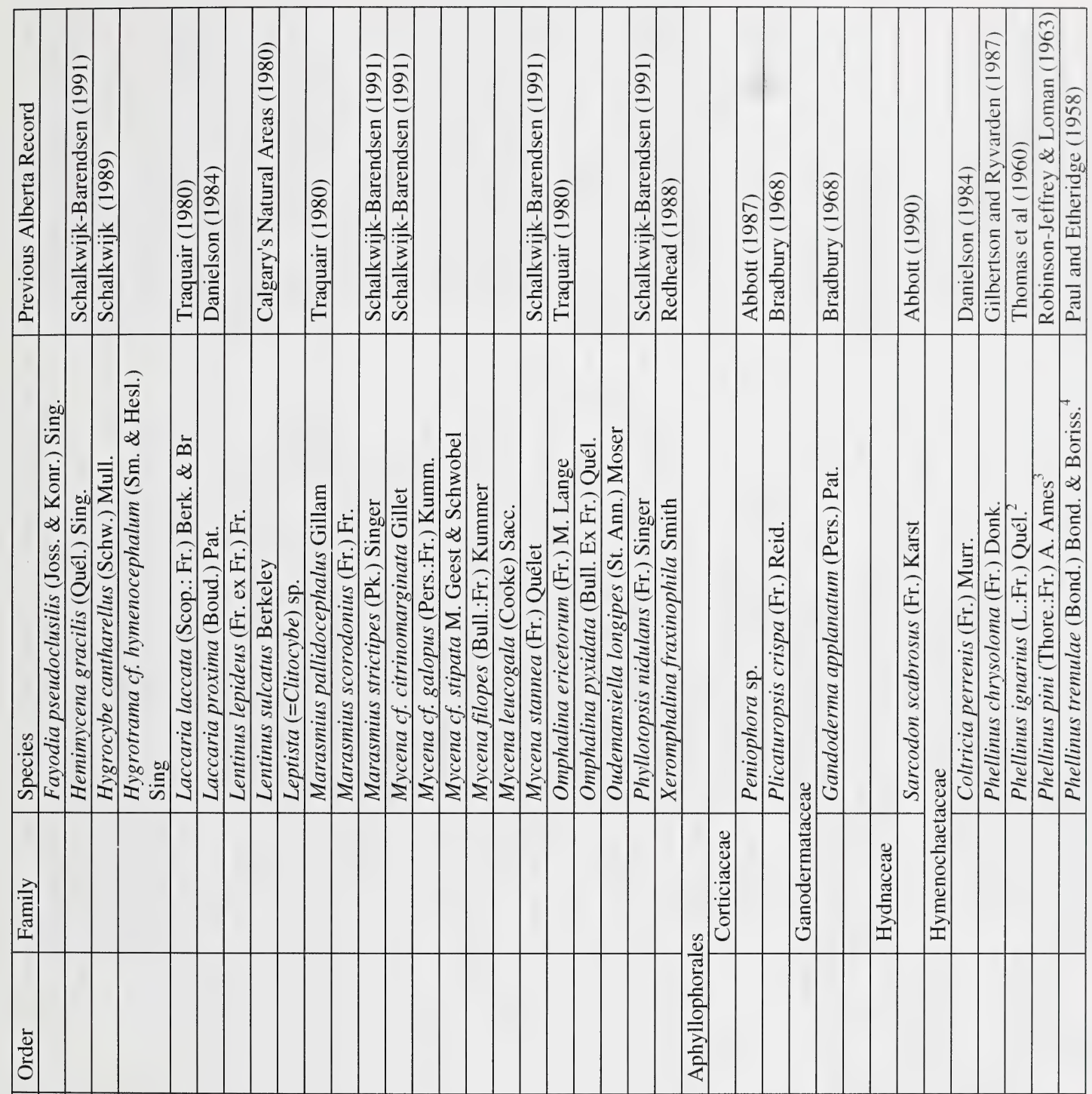

ฮิ

0
0
$: 0$
$\vdots 0$
0
0
0
0

อี 

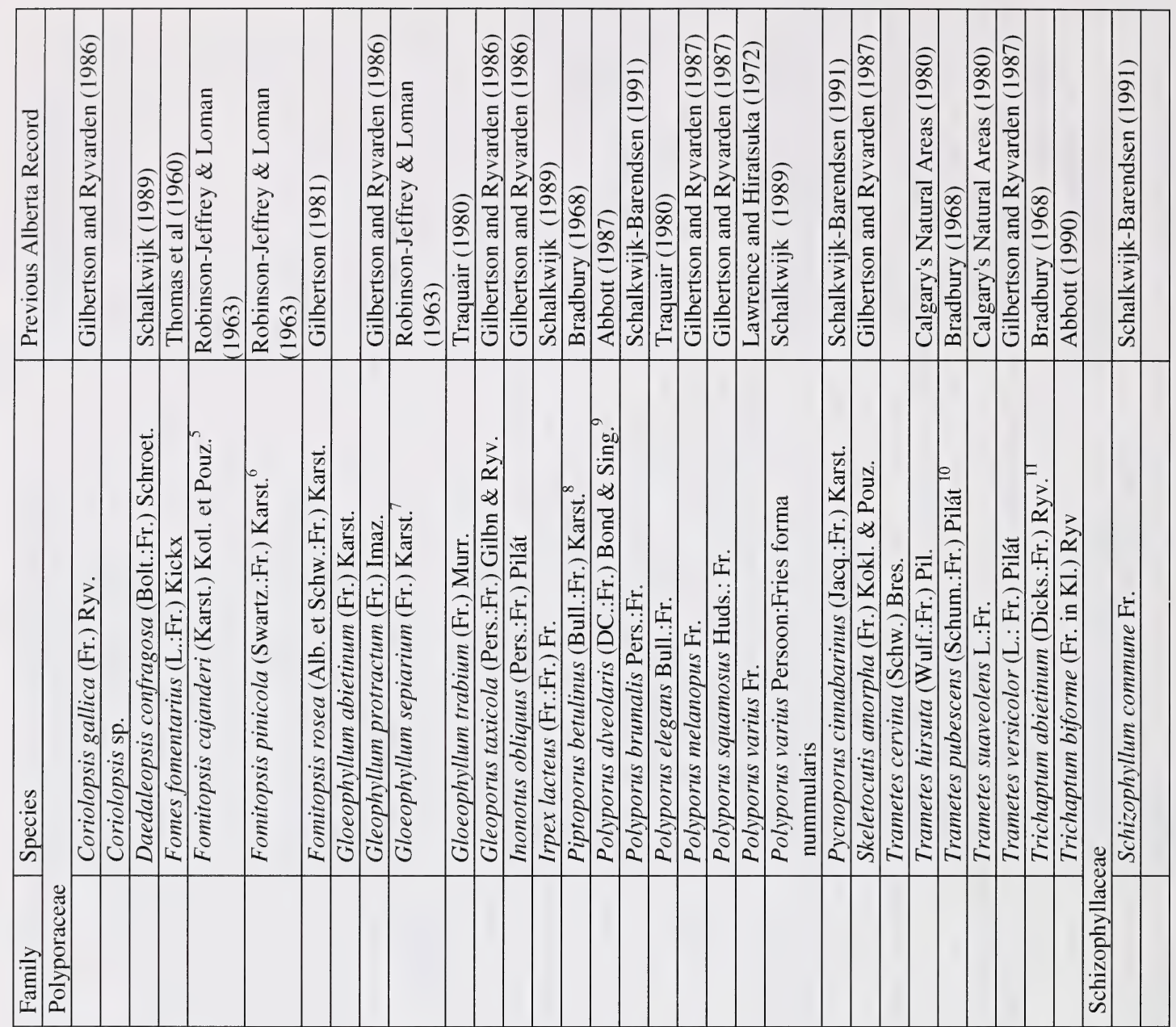

홓 

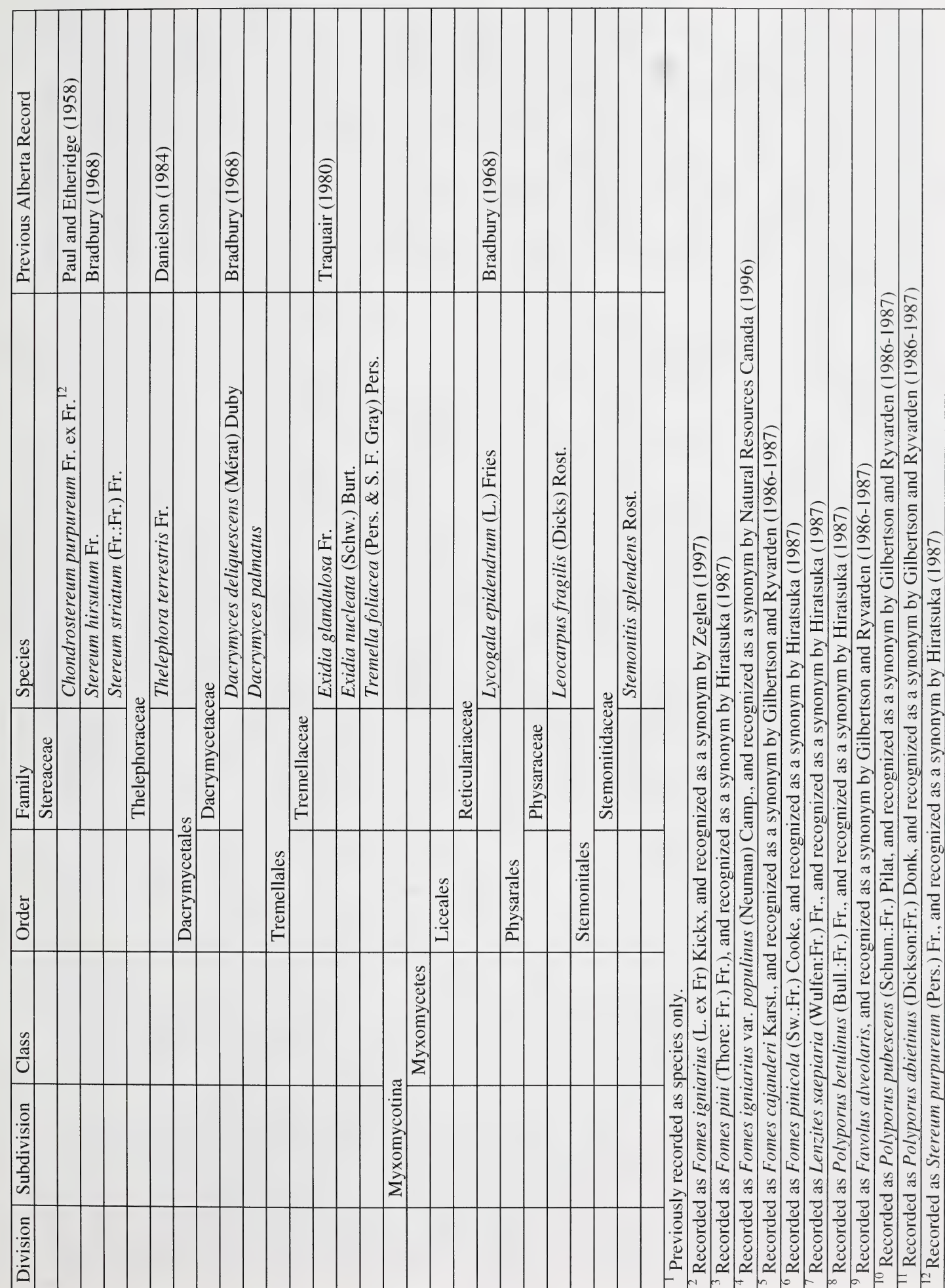
Appendix 2. Annotated species list for La Butte Creek Wildland

\begin{tabular}{|c|c|c|c|c|c|c|}
\hline Species & Habitat & $\begin{array}{l}\text { NAD } \\
83\end{array}$ & Easting & Northing & Date & $\begin{array}{l}\text { Specimen } \\
\text { No. }\end{array}$ \\
\hline $\begin{array}{l}\text { (? Inocybe sp.) infected } \\
\text { with Cladosporium sp }\end{array}$ & on moss with black spruce & $12 \mathrm{~V}$ & 485975.90 & 6581957.42 & July 152001 & LBC107a \\
\hline $\begin{array}{l}\text { Chondrostereum } \\
\text { purpureum }\end{array}$ & on dead aspen poplar bark & $12 \mathrm{~V}$ & 491127.72 & 6580205.28 & July 82001 & LBC032 \\
\hline Clitocybe truncicola & in moss on rotted log & $12 \mathrm{~V}$ & 477088.94 & 6584366.43 & July 142001 & LBC070 \\
\hline Collybia cf. dryophila & on moss in spruce bog & $12 \mathrm{~V}$ & 492236.38 & 6580251.83 & July 122001 & LBC097 \\
\hline Collybia cf. dryophila & on moss in spruce bog & $12 \mathrm{~V}$ & 492236.38 & 6580251.83 & July 122001 & LBC098 \\
\hline Collybia dryophila & $\begin{array}{l}\text { on ground in moss in willow } \\
\text { forest }\end{array}$ & $12 \mathrm{~V}$ & 474464.27 & 6587728.02 & July 62001 & LBC011 \\
\hline Collybia dryophila & on moss in white spruce forest & $12 \mathrm{~V}$ & 474126.65 & 6585095.84 & July 72001 & LBC029 \\
\hline Collybia dryophila & on aspen poplar leaves & $12 \mathrm{~V}$ & 491127.72 & 6580205.28 & July 82001 & LBC034 \\
\hline Collybia dryophila & on moss in aspen forest & $12 \mathrm{~V}$ & 491127.72 & 6580205.28 & July 82001 & LBC035 \\
\hline Collybia dryophila & $\begin{array}{l}\text { in moss of white spruce /aspen } \\
\text { forest }\end{array}$ & $12 \mathrm{~V}$ & 491127.72 & 6580205.28 & July 82001 & LBC036 \\
\hline Collybia dryophila & in moss in black spruce bog & $12 \mathrm{~V}$ & 485975.90 & 6581957.42 & July 102001 & LBC044 \\
\hline Collybia dryophila & $\begin{array}{l}\text { with white spruce and balsam } \\
\text { poplar }\end{array}$ & $12 \mathrm{~V}$ & 475891.84 & 6580230.43 & July 112001 & LBC088a \\
\hline Collybia dryophila & in white spruce stand & $12 \mathrm{~V}$ & 477499.07 & 6584378.30 & July 142001 & LBC091 \\
\hline Collybia marasmioides & $\begin{array}{l}\text { on ground in moss in willow } \\
\text { forest }\end{array}$ & $12 \mathrm{~V}$ & 474464.27 & 6587728.02 & July 62001 & LBC010 \\
\hline Coprinus hemerobius & on mud of beaverdam & $12 \mathrm{~V}$ & 489206.86 & 6580689.96 & July 72001 & LBC016b \\
\hline Coprinus micaceus & on equisetum sp. & $12 \mathrm{~V}$ & 489206.86 & 6580689.96 & July 72001 & LBC016 \\
\hline Coprinus micaceus & on moss leaves under willow & $12 \mathrm{~V}$ & 474853.33 & 65841.35 & July 62001 & LBC016a \\
\hline Coriolopsis gallica & $\begin{array}{l}\text { on fallen aspen with bark } \\
\text { remaining }\end{array}$ & $12 \mathrm{~V}$ & 474853.33 & 6585541.35 & July 72001 & LBC082 \\
\hline Coriolopsis sp. & $\begin{array}{l}\text { on fallen aspen with bark } \\
\text { remaining }\end{array}$ & $12 \mathrm{~V}$ & 474853.33 & 6585541.35 & July 72001 & LBC120 \\
\hline Cortinarius cf. huronensis & [with moss] & $12 \mathrm{~V}$ & 478813.83 & 6579354.52 & July 152001 & LBC106 \\
\hline Cortinarius cf. huronensis & on moss with black spruce & $12 \mathrm{~V}$ & 485975.90 & 6581957.42 & July 152001 & LBC107 \\
\hline Cortinarius cf. privignus & $\begin{array}{l}\text { in duff of white spruce/aspen } \\
\text { poplar stand }\end{array}$ & $12 \mathrm{~V}$ & 477499.07 & 6584378.30 & July 142001 & LBC067 \\
\hline $\begin{array}{l}\text { Cortinarius cf. } \\
\text { raphanoides }\end{array}$ & on moss in black spruce bog & $12 \mathrm{~V}$ & 492236.38 & 6580251.83 & July 122001 & LBC094 \\
\hline Cortinarius cf. vespertinus & in moss in black spruce bog & $12 \mathrm{~V}$ & 485975.90 & 6581957.42 & July 102001 & LBC043 \\
\hline Cortinarius citrinifolius & {$[?]$} & $12 \mathrm{~V}$ & 492236.38 & 6580251.83 & July 122001 & LBC093 \\
\hline Cortinarius mисоsиs & $\begin{array}{l}\text { on game trail in aspen forest, } \\
\text { with grasses, bearberry \& } \\
\text { vetch }\end{array}$ & $12 \mathrm{~V}$ & 474126.65 & 6585095.84 & July 72001 & LBC023 \\
\hline Cortinarius trivialis & in moss in alder forest & $12 \mathrm{~V}$ & 474126.65 & 6585095.84 & July 72001 & LBC024 \\
\hline
\end{tabular}




\begin{tabular}{|c|c|c|c|c|c|c|}
\hline Species & Habitat & $\begin{array}{l}\text { NAD } \\
83\end{array}$ & Easting & Northing & Date & $\begin{array}{l}\text { Specimen } \\
\text { No. }\end{array}$ \\
\hline Crepidotus ellipsoideus & on dead alder & $12 \mathrm{~V}$ & 486095.89 & 6582093.18 & July 102001 & LBC050 \\
\hline Crepidotus ellipsoideus & on alder/old, dry & $12 \mathrm{~V}$ & 477499.07 & 6584378.30 & July 102001 & LBC059 \\
\hline Crepidotus ellipsoideus & on wood & $12 \mathrm{~V}$ & 477499.07 & 6584378.30 & July 142001 & LBC092 \\
\hline Crepidotus mollis & on dead wood & $12 \mathrm{~V}$ & 477783.17 & 6584510.43 & July 142001 & LBC063 \\
\hline Dacrymyces palmatus & $\begin{array}{l}\text { on birch and alder/specimen } \\
\text { missing }\end{array}$ & $12 \mathrm{~V}$ & 486095.89 & 6582093.18 & July 102001 & LBC048 \\
\hline Dacrymyces deliquescens & on rotten balsam poplar & $12 \mathrm{~V}$ & 474126.65 & 6585095.84 & July 72001 & LBC030 \\
\hline Daedaleopsis confragosa & $\begin{array}{l}\text { on older fire killed log in black } \\
\text { spruce bog }\end{array}$ & $12 \mathrm{~V}$ & 485975.90 & 6581957.42 & July 102001 & LBC040 \\
\hline Daedaleopsis confragosa & on white birch twig & $12 \mathrm{~V}$ & 474853.33 & 6585541.35 & July 72001 & LBC076 \\
\hline Exidia glandulosa & on alder & $12 \mathrm{~V}$ & 477088.94 & 6584366.43 & July 142001 & LBC074 \\
\hline Exidia nucleata & on dead alder & $12 \mathrm{~V}$ & 486095.89 & 6582093.18 & July 102001 & LBC122 \\
\hline Fayodia pseudoclusilis & $\begin{array}{l}\text { on partly decomposed willow } \\
\text { leaves }\end{array}$ & $12 \mathrm{~V}$ & 474464.27 & 6587728.02 & July 62001 & $\mathrm{LBC} 012$ \\
\hline Fomes fomentarius & on white birch & $12 \mathrm{~V}$ & 474859.30 & 6587445.19 & July 62001 & LBC003 \\
\hline Fomes fomentarius & on birch & $12 \mathrm{~V}$ & 474919.40 & 6587555.91 & July 62001 & LBC008 \\
\hline Fomes fomentarius & on alder & $12 \mathrm{~V}$ & 477499.07 & 6584378.30 & July 102001 & LBC119 \\
\hline Fomitopsis cajanderi & on rotting balsam poplar log & $12 \mathrm{~V}$ & 474919.40 & 6587555.91 & July 62001 & LBC006a \\
\hline Fomitopsis pinicola & on dead spruce log & $12 \mathrm{~V}$ & 481025.28 & 6582607.45 & July 92001 & LBC039 \\
\hline Fomitopsis pinicola & on spruce stump & $12 \mathrm{~V}$ & 474853.33 & 6585541.35 & July 72001 & LBC078 \\
\hline Gandoderma applanatum & on wood & $12 \mathrm{~V}$ & 474729.26 & 6587026.10 & July 62001 & LBC004 \\
\hline Gleophyllum sepiarium & on dead wood & $12 \mathrm{~V}$ & 486112.51 & 6581970.06 & July 102001 & LBC053 \\
\hline Gleophyllum sepiarium & on white spruce & $12 \mathrm{~V}$ & 499258.40 & 6579539.65 & July 152001 & LBC108 \\
\hline Gleophyllum trabium & on fire killed pine & $12 \mathrm{~V}$ & 485975.90 & 6581957.42 & July 102001 & LBC046 \\
\hline Gymnopilus cf. humicola & on wood & $12 \mathrm{~V}$ & 477088.94 & 6584366.43 & July 142001 & LBC071 \\
\hline Hemimycena gracilis & on moss in white spruce forest & $12 \mathrm{~V}$ & 489206.86 & 6580689.96 & July 82001 & LBC026 \\
\hline Hygrocybe cantharellus & in sphagnum & $12 \mathrm{~V}$ & 493979.68 & 6577631.01 & July 152001 & LBC111 \\
\hline $\begin{array}{l}\text { Hygrophoropsis } \\
\text { aurantiaca }\end{array}$ & $\begin{array}{l}\text { on rotten log in swamp with } \\
\text { calla and alder }\end{array}$ & $12 \mathrm{~V}$ & 475891.84 & 6580230.43 & July 112001 & LBC086 \\
\hline \begin{tabular}{|l|} 
Hygrotrama cf. \\
hymenocephalum \\
\end{tabular} & on sphagnum & $12 \mathrm{~V}$ & 493979.68 & 6577631.01 & July 152001 & LBC109 \\
\hline Inocybe cf. calamistrata & on willow & $12 \mathrm{~V}$ & 492236.38 & 6580251.83 & July 122001 & LBC095 \\
\hline Inocybe cf. fastigata & $\begin{array}{l}\text { on sphagnum in black spruce } \\
\text { bog }\end{array}$ & $12 \mathrm{~V}$ & 486112.51 & 6581970.06 & July 102001 & LBC058 \\
\hline Inocybe cf. frigidula & $\begin{array}{l}\text { on ground in mixed white } \\
\text { spruce/aspen stand }\end{array}$ & $12 \mathrm{~V}$ & 492236.38 & 6580251.83 & July 122001 & LBC096 \\
\hline Inocybe cf. gymnocarpa & in saline meadow with willow & $12 \mathrm{~V}$ & 478813.83 & 6579354.52 & July 152001 & LBC116 \\
\hline Inocybe cf. petiginosa & $\begin{array}{l}\text { in moss in black spruce } \\
\text { swamp }\end{array}$ & $12 \mathrm{~V}$ & 485975.90 & 6581957.42 & July 102001 & LBC045 \\
\hline
\end{tabular}




\begin{tabular}{|c|c|c|c|c|c|c|}
\hline Species & Habitat & $\begin{array}{l}\text { NAD } \\
83\end{array}$ & Easting & Northing & Date & $\begin{array}{l}\text { Specimen } \\
\text { No. }\end{array}$ \\
\hline Inocybe mixtilis & in moss with black spruce & $12 \mathrm{~V}$ & 486112.51 & 6581970.06 & July 102001 & LBC055 \\
\hline Irpex lacteus & on bark of dead alder & $12 \mathrm{~V}$ & 491127.72 & 6580205.28 & July 82001 & LBC033 \\
\hline Irpex lacteus & on wood & $12 \mathrm{~V}$ & 477499.07 & 6584378.30 & July 152001 & LBC102 \\
\hline Leccinum insigne & $\begin{array}{l}\text { on edge of aspen stand and } \\
\text { black spruce bog }\end{array}$ & $12 \mathrm{~V}$ & 486112.51 & 6581970.06 & July 102001 & LBC037 \\
\hline Leccinum scabrum & $\begin{array}{l}\text { on bare ground on shoreline of } \\
\text { La Butte Creek }\end{array}$ & $12 \mathrm{~V}$ & 477783.17 & 6584510.43 & July 142001 & LBC062 \\
\hline Lentinus lepideus & $\begin{array}{l}\text { on dead/dry large driftwood } \\
\text { logs }\end{array}$ & $12 \mathrm{~V}$ & 474016.20 & 6585200.54 & July 72001 & LBC051 \\
\hline Lentinus lepideus & $\begin{array}{l}\text { on fire killed black spruce, in } \\
\text { area with Labrador tea \& wild } \\
\text { red raspberry }\end{array}$ & $12 \mathrm{~V}$ & 474853.33 & 6585541.35 & July 72001 & LBC081 \\
\hline Lentinus sulcatus & on dead/dry wood & $12 \mathrm{~V}$ & 474853.33 & 6585541.35 & July 72001 & LBC123 \\
\hline Leocarpus fragilis & on lichen in moss & $12 \mathrm{~V}$ & 477499.07 & 6584378.30 & July 142001 & LBC069 \\
\hline Lycogala epidendrum & on wood & $12 \mathrm{~V}$ & 477499.07 & 6584378.30 & July 142001 & LBC068 \\
\hline $\begin{array}{l}\text { Marasmius } \\
\text { pallidocephalus }\end{array}$ & $\begin{array}{l}\text { in moss in white spruce/white } \\
\text { birch forest }\end{array}$ & $12 \mathrm{~V}$ & 475063.66 & 6586400.20 & July 72001 & $\mathrm{LBC} 020$ \\
\hline \begin{tabular}{|l|} 
Marasmius \\
pallidocephalus
\end{tabular} & $\begin{array}{l}\text { on bits of spruce cone \& } \\
\text { needles }\end{array}$ & $12 \mathrm{~V}$ & 474126.65 & 6585095.84 & July 72001 & LBC028 \\
\hline Marasmius scorodonius & in sphagnum & $12 \mathrm{~V}$ & 493979.68 & 6577631.01 & July 152001 & LBC114 \\
\hline Marasmius strictipes & $\begin{array}{l}\text { on wood in white spruce } \\
\text { forest-willow area }\end{array}$ & $12 \mathrm{~V}$ & 477499.07 & 6584378.30 & July 142001 & LBC060 \\
\hline Melastiza chateri & in moss on mud of beaverdam & $12 \mathrm{~V}$ & 489206.86 & 6580689.96 & July 82001 & LBC038 \\
\hline $\begin{array}{l}\text { Mycena cf. } \\
\text { citrinomarginata }\end{array}$ & $\begin{array}{l}\text { in moss in white spruce/white } \\
\text { birch forest }\end{array}$ & $12 \mathrm{~V}$ & 475063.66 & 6586400.20 & July 72001 & LBC021 \\
\hline Mycena cf. stipata & {$[?]$} & $12 \mathrm{~V}$ & 486112.51 & 6581970.06 & July 102001 & LBC057 \\
\hline Omphalina ericetorum & on sphagnum in spruce bog & $12 \mathrm{~V}$ & 485975.90 & 6581957.42 & July 102001 & LBC047 \\
\hline Omphalina ericetorum & $\begin{array}{l}\text { on moss in black spruce with } \\
\text { sphagnum and rushes }\end{array}$ & $12 \mathrm{~V}$ & 486112.51 & 6581970.06 & July 102001 & LBC052 \\
\hline Omphalina ericetorum & in sphagnum & $12 \mathrm{~V}$ & 493979.68 & 6577631.01 & July 152001 & LBC117 \\
\hline Oudemansiella longipes & on wood in young aspen stand & $12 \mathrm{~V}$ & 493979.68 & 6577631.01 & July 152001 & LBC1 18 \\
\hline Oudemansiella longipes & in moss with alder & $12 \mathrm{~V}$ & 478813.83 & 6579354.52 & July 152001 & LBC105 \\
\hline Oudemansiella longipes & in moss with alder & $12 \mathrm{~V}$ & 478813.83 & 6579354.52 & July 152001 & LBC105a \\
\hline Peniophora sp. & on old spruce & $12 \mathrm{~V}$ & 499258.40 & 6579539.65 & July 152001 & LBC115 \\
\hline Phellinus pini & on old wood & $12 \mathrm{~V}$ & 477499.07 & 6584378.30 & July 152001 & LBC101 \\
\hline Phellinus tremulae & $\begin{array}{l}\text { one meter from ground on } \\
\text { dead balsam poplar }\end{array}$ & $12 \mathrm{~V}$ & 474919.40 & 6587555.91 & July 72001 & LBC075 \\
\hline Phellinus tremulae & on aspen poplar & $12 \mathrm{~V}$ & 474853.33 & 6585541.35 & July 72001 & LBC077 \\
\hline Phyllotopsis nidulans & on dead spruce branch & $12 \mathrm{~V}$ & 477499.07 & 6584378.30 & July 142001 & LBC066 \\
\hline Piptoporus betulinus & on dead birch & $12 \mathrm{~V}$ & 474853.33 & 6585541.35 & July 72001 & LBC079 \\
\hline
\end{tabular}




\begin{tabular}{|c|c|c|c|c|c|c|}
\hline Species & Habitat & $\begin{array}{l}\text { NAD } \\
83\end{array}$ & Easting & Northing & Date & $\begin{array}{l}\text { Specimen } \\
\text { No. }\end{array}$ \\
\hline Piptoporus betulinus & on dead birch & $12 \mathrm{~V}$ & 474853.33 & 6585541.35 & July 72001 & LBC080 \\
\hline Pleurotus ostreatus & on balsam poplar & $12 \mathrm{~V}$ & 474729.26 & 6587026.10 & July 62001 & LBCOO2 \\
\hline Plicaturopsis crispa & on birch & $12 \mathrm{~V}$ & 474919.40 & 6587555.91 & July 62001 & LBCOO9 \\
\hline Plicaturopsis crispa & on alder & $12 \mathrm{~V}$ & 475891.84 & 6580230.43 & July 112001 & LBC083 \\
\hline Plicaturopsis crispa & on alder/ old, dry & $12 \mathrm{~V}$ & 477499.07 & 6584378.30 & July 102001 & $\mathrm{LBC} 121$ \\
\hline Pluteus cervinus & on rotting balsam poplar log & $12 \mathrm{~V}$ & 474919.40 & 6587555.91 & July 62001 & LBC006 \\
\hline Pluteus pestatus & $\begin{array}{l}\text { growing out the end of a rotted } \\
\text { birch log }\end{array}$ & $12 \mathrm{~V}$ & 489206.86 & 6580689.96 & July 82001 & LBC025 \\
\hline Polyporus alveolaris & on aspen & $12 \mathrm{~V}$ & 477783.17 & 6584510.43 & July 142001 & LBC061a \\
\hline Polyporus elegans & on alder & $12 \mathrm{~V}$ & 477499.07 & 6584378.30 & July 142001 & LBC090 \\
\hline Polyporus squamosus & on rotted stump & $12 \mathrm{~V}$ & 477499.07 & 6584378.30 & July 142001 & LBC061 \\
\hline Polyporus varius & $\begin{array}{l}\text { on birch and rotting balsam } \\
\text { poplar }\end{array}$ & $12 \mathrm{~V}$ & 474919.40 & 6587555.91 & July 62001 & LBC007 \\
\hline Polyporus varius & on balsam poplar & $12 \mathrm{~V}$ & 486112.51 & 6581970.06 & July 102001 & LBC054 \\
\hline $\begin{array}{l}\text { Polyporus varius forma } \\
\text { nummularis }\end{array}$ & on dead wood & $12 \mathrm{~V}$ & 475891.84 & 6580230.43 & July 112001 & LBC085 \\
\hline Psathyrella cf. gracilis & $\begin{array}{l}\text { on leaf litter amongst alder at } \\
\text { creek edge }\end{array}$ & $12 \mathrm{~V}$ & 482191.49 & 6582371.79 & July 152001 & LBC 104 \\
\hline Psathyrella cf. velutina & on ground in alder flat & $12 \mathrm{~V}$ & 475063.66 & 6586400.20 & July 72001 & LBC018 \\
\hline Psathyrella cf. velutina & on ground in alder flat & $12 \mathrm{~V}$ & 475063.66 & 6586400.20 & July 72001 & LBC019 \\
\hline Psathyrella subnuda & on moss in white spruce stand & $12 \mathrm{~V}$ & 475891.84 & 6580230.43 & July 112001 & LBC084 \\
\hline Psathyrella subnuda & $\begin{array}{l}\text { on ground in open dry } \\
\text { meadow with aspen and } \\
\text { willow }\end{array}$ & $12 \mathrm{~V}$ & 478813.83 & 6579354.52 & July 152001 & LBC 113 \\
\hline Pycnoporus cinnabarinus & on dead alder & $12 \mathrm{~V}$ & 486112.51 & 6581970.06 & July 102001 & LBC041 \\
\hline Rhodocybe caelata & [?] & $12 \mathrm{~V}$ & 475891.84 & 6580230.43 & July 112001 & LBC087 \\
\hline Rhodocybe caelata $C f$. & in black spruce bog & $12 \mathrm{~V}$ & 492236.38 & 6580251.83 & July 122001 & LBC 100 \\
\hline Russula cf. fragilis & $\begin{array}{l}\text { on alder bark in balsam poplar } \\
\text { forest }\end{array}$ & $12 \mathrm{~V}$ & 492236.38 & 6580251.83 & July 122001 & LBC099 \\
\hline Russula emetica & $\begin{array}{l}\text { on sphagnum in wet } \\
\text { birch/alder swamp }\end{array}$ & $12 \mathrm{~V}$ & 493979.68 & 6577631.01 & July 152001 & $\mathrm{LBC} 110$ \\
\hline Russula fragilis & in alder swamp & $12 \mathrm{~V}$ & 474126.65 & 6585095.84 & July 72001 & LBC022 \\
\hline Russula fragilis & in white spruce stand & $12 \mathrm{~V}$ & 477499.07 & 6584378.30 & July 142001 & LBC089 \\
\hline Russula fragilis & on mossy log & $12 \mathrm{~V}$ & 477499.07 & 6584378.30 & July 152001 & LBC 103 \\
\hline Russula vesca & $\begin{array}{l}\text { on the shoreline of recently } \\
\text { drawn down La Butte Creek }\end{array}$ & $12 \mathrm{~V}$ & 477783.17 & 6584510.43 & July 142001 & LBC064 \\
\hline Sarcodon scabrosus & under aspen /specimen moldy & $12 \mathrm{~V}$ & 477499.07 & 6584378.30 & July 142001 & LBC073 \\
\hline Schizophyllum commune & on dead wood & $12 \mathrm{~V}$ & 485975.90 & 6581957.42 & July 102001 & LBC042 \\
\hline Scutellinia scutellata & on mud of beaverdam & $12 \mathrm{~V}$ & 489206.86 & 6580689.96 & July 82001 & LBC031 \\
\hline Stereum hirsutum & rotted birch log & $12 \mathrm{~V}$ & 474859.30 & 6587445.19 & July 62001 & LBC005 \\
\hline Trametes pubescens & on wood & $12 \mathrm{~V}$ & 486112.51 & 6581970.06 & July 102001 & LBC056 \\
\hline Tremella foliacea & on dead alder & $12 \mathrm{~V}$ & 486095.89 & 6582093.18 & July 102001 & LBC049 \\
\hline Tremella foliacea & on alder & $12 \mathrm{~V}$ & 475891.84 & 6580230.43 & July 112001 & LBC083a \\
\hline Trichaptum biforme & on birch & $12 \mathrm{~V}$ & 474919.40 & 6587555.91 & July 62001 & LBC013 \\
\hline Tubaria furfuracea & $\begin{array}{l}\text { on dead wood with moss in } \\
\text { white spruce forest }\end{array}$ & $12 \mathrm{~V}$ & 475891.84 & 6580230.43 & July 112001 & LBC088 \\
\hline Tubaria pellucida & [?] & $12 \mathrm{~V}$ & 475063.66 & 6586400.20 & July 72001 & LBC017 \\
\hline
\end{tabular}




\section{Appendix 3. Annotated species list for Fidler-Greywillow Wildland}

\begin{tabular}{|c|c|c|c|c|c|c|}
\hline Species & Habitat & $\begin{array}{l}\text { NAD } \\
83\end{array}$ & Easting & Northing & Date & $\begin{array}{l}\text { Specimen } \\
\text { No. }\end{array}$ \\
\hline Agaricus bernardii & on sand near balsam poplar & $12 \mathrm{~V}$ & 494503 & 6513031 & July 242001 & FG070 \\
\hline Armillaria mellea & on ground & $12 \mathrm{~V}$ & 547141 & 6565461 & July 202001 & FG037 \\
\hline Astraeus hygrometricus & on sand at upper beach level & $12 \mathrm{~V}$ & 532885.51 & 6552112.57 & July 182001 & FG014 \\
\hline Astraeus hygrometricus & in sand 2-4 cm deep & $12 \mathrm{~V}$ & 547141 & 6565461 & July 202001 & FG035 \\
\hline Astraeus hygrometricus & on sand of upper beach & $12 \mathrm{~V}$ & 521564.95 & 6532104.53 & July 212001 & FG041 \\
\hline Bisporella citrina & on rotting stick & $12 \mathrm{~V}$ & 535656.32 & 6554192.38 & July 192001 & FG024 \\
\hline Collybia dryophila & on moss in spruce forest & $12 \mathrm{~V}$ & 535131.81 & 6553858.37 & July 192001 & FG019 \\
\hline Collybia dryophila & on feather/feather moss & $12 \mathrm{~V}$ & 535483.64 & 6554269.03 & July 202001 & FG031 \\
\hline Collybia dryophila & on moss & $12 \mathrm{~V}$ & 535483.64 & 6554269.03 & July 202001 & FG033 \\
\hline Coltricia perrenis & on sand with reindeer lichen & $12 \mathrm{~V}$ & 536660 & 6554586 & July 192001 & FG015 \\
\hline Coltricia perrenis & on humus on sand & $12 \mathrm{~V}$ & 547141 & 6565461 & July 202001 & FG040 \\
\hline Coltricia perrenis & $\begin{array}{l}\text { on sand along path, with } \\
\text { lichen and blueberry }\end{array}$ & $12 \mathrm{~V}$ & 536643 & 6555716 & July 222001 & FG049 \\
\hline Coltricia perrenis & on sand & $12 \mathrm{~V}$ & 514758 & 6517473 & July 242001 & FG061 \\
\hline Coriolopsis gallica & on fire killed pine & $12 \mathrm{~V}$ & 547141 & 6565461 & July 202001 & FG036 \\
\hline Cortinarius armillatus & $\begin{array}{l}\text { on ground in aspen/balsam } \\
\text { poplar stand }\end{array}$ & $12 \mathrm{~V}$ & 509615 & 6520911 & July 242001 & FG069 \\
\hline $\begin{array}{l}\text { Cortinarius brunneus var. } \\
\text { grandicolor }\end{array}$ & on moss with black spruce & $12 \mathrm{~V}$ & 535131.81 & 6553858.37 & July 192001 & FG020 \\
\hline $\begin{array}{l}\text { Cortinarius brunneus var. } \\
\text { grandicolor }\end{array}$ & on sand at lake edge & $12 \mathrm{~V}$ & 529525 & 6553460 & July 232001 & FG053 \\
\hline Cortinarius cf. casimiri & $\begin{array}{l}\text { on sand with rushes at spray } \\
\text { line of lake }\end{array}$ & $12 \mathrm{~V}$ & 536660 & 6554586 & July 192001 & FG017 \\
\hline Cortinarius cf. casimiri & $\begin{array}{l}\text { on sand with rushes at spray } \\
\text { line of lake }\end{array}$ & $12 \mathrm{~V}$ & 535131.81 & 6553858.37 & July 192001 & FG018 \\
\hline Cortinarius cf. jubarinus & on sphagnum & $12 \mathrm{~V}$ & 535483.64 & 6554269.03 & July 202001 & FG032 \\
\hline Cortinarius helobius & on sand at lake edge & $12 \mathrm{~V}$ & 529525 & 6553460 & July 232001 & FG053 \\
\hline Daedaleopsis confragosa & on balsam poplar & $12 \mathrm{~V}$ & 529525 & 6553460 & July 232001 & FG056 \\
\hline Dermocybe cinnamomeobadia & in sphagnum & $12 \mathrm{~V}$ & 509615 & 6520911 & July 242001 & FG063 \\
\hline Dermocybe uliginosa & in moss on open bog & $12 \mathrm{~V}$ & 535213.77 & 6553895.56 & July 182001 & FG010 \\
\hline Fomes fomentarius & on birch & $12 \mathrm{~V}$ & 532787.66 & 6552523.92 & July 172001 & FG008 \\
\hline Fomes fomentarius & on birch & $12 \mathrm{~V}$ & 533249 & 6552781 & July 262001 & FG071 \\
\hline Fomitopsis pinicola & on black spruce and jackpine & $12 \mathrm{~V}$ & 535131.81 & 6553858.37 & July 182001 & FG012 \\
\hline Fomitopsis pinicola & on dead black spruce & $12 \mathrm{~V}$ & 534057 & 6553874 & July 222001 & FG051 \\
\hline Fomitopsis pinicola & on balsam poplar & $12 \mathrm{~V}$ & 509615 & 6520911 & July 242001 & FG065 \\
\hline Galerina hypnorum & on moss in black spruce stand & $12 \mathrm{~V}$ & 532688.21 & 6552404.78 & July 172001 & FG002 \\
\hline Galerina paludosa & on moss with black spruce & $12 \mathrm{~V}$ & 535131.81 & 6553858.37 & July 192001 & FG022 \\
\hline Galerina paludosa & $\begin{array}{l}\text { on leaf litter in black } \\
\text { spruce/aspen }\end{array}$ & $12 \mathrm{~V}$ & 535092.25 & 6553951.79 & July 192001 & FG026 \\
\hline Galerina paludosa & in sphagnum & $12 \mathrm{~V}$ & 535483.64 & 6554269.03 & July 202001 & FG030 \\
\hline Gandoderma applanatum & on wood & $12 \mathrm{~V}$ & 533249 & 6552781 & July 262001 & FG072 \\
\hline Gandoderma applanatum & on wood & $12 \mathrm{~V}$ & 533249 & 6552781 & July 262001 & FG073 \\
\hline Gloeophyllum abietinum & on dead black spruce & $12 \mathrm{~V}$ & 537833 & 6555131 & July 222001 & FG046 \\
\hline Gymnopilus humicola & on birch & $12 \mathrm{~V}$ & 535131.81 & 6553858.37 & July 192001 & FG021 \\
\hline
\end{tabular}




\begin{tabular}{|c|c|c|c|c|c|c|}
\hline Species & Habitat & $\begin{array}{l}\text { NAD } \\
83\end{array}$ & Easting & Northing & Date & $\begin{array}{l}\text { Specimen } \\
\text { No. }\end{array}$ \\
\hline Hebeloma cf. velatum & edge of pine and creek & $12 \mathrm{~V}$ & 535656.32 & 6554192.38 & July 192001 & FG027 \\
\hline Inocybe cf. frigidula & on sand & $12 \mathrm{~V}$ & 534970.30 & 6553653.18 & July 202001 & FG028 \\
\hline Laccaria laccata & $\begin{array}{l}\text { on leaf litter in black } \\
\text { spruce/aspen }\end{array}$ & $12 \mathrm{~V}$ & 535483.64 & 6554269.03 & July 202001 & FG029 \\
\hline Laccaria proxima & on sand & $12 \mathrm{~V}$ & 537833 & 6555131 & July 222001 & FG047 \\
\hline Lactarius cf. obscuratus & in leaf litter & $12 \mathrm{~V}$ & 547141 & 6565461 & July 202001 & FG038 \\
\hline Lactarius fragilis & $\begin{array}{l}\text { in shade of upturned stump, } \\
\text { on moss in sand }\end{array}$ & $12 \mathrm{~V}$ & 534057 & 6553874 & July 222001 & FG050 \\
\hline Leccinum insigne & $\begin{array}{l}\text { with aspen poplar, occasional } \\
\text { birch and balsam poplar }\end{array}$ & $12 \mathrm{~V}$ & 509615 & 6520911 & July 242001 & FG064 \\
\hline Leccinum scabrum & $\begin{array}{l}\text { in moss under } \\
\text { aspen/birch/white spruce }\end{array}$ & $12 \mathrm{~V}$ & 529525 & 6553460 & July 232001 & FG054 \\
\hline Leptista $(=$ Clitocybe $) s p$. & on moss & $12 \mathrm{~V}$ & 535213.77 & 6553895.56 & July 182001 & FG013 \\
\hline Mycena stannea & on moss & $12 \mathrm{~V}$ & 547141 & 6565461 & July 202001 & FG039 \\
\hline Omphalina ericetorum & $\begin{array}{l}\text { in spruce bog with liverworts, } \\
\text { sphagnum \& other mosses }\end{array}$ & $12 \mathrm{~V}$ & 532885.51 & 6552112.57 & July 172001 & FG001 \\
\hline Omphalina ericetorum & $\begin{array}{l}\text { on moss with black spruce } \\
\text { (with FG022 - Galerina } \\
\text { paludosa Fr.) }\end{array}$ & $12 \mathrm{~V}$ & 535131.81 & 6553858.37 & July 192001 & FG074 \\
\hline Omphalina pyxidata & on moss in black spruce stand & $12 \mathrm{~V}$ & 532688.21 & 6552404.78 & July 172001 & FG006 \\
\hline Peziza badia & \begin{tabular}{|l} 
growing on sand with moss \& \\
liverworts
\end{tabular} & $12 \mathrm{~V}$ & 536660 & 6554586 & July 192001 & FG016 \\
\hline Phellinus tremulae & on balsam poplar & $12 \mathrm{~V}$ & 509615 & 6520911 & July 242001 & FG066 \\
\hline Piptoporus betulinus & on birch & $12 \mathrm{~V}$ & 509615 & 6520911 & July 242001 & FG068 \\
\hline Pluteus cervinus & on birch log & $12 \mathrm{~V}$ & 532688.21 & 6552404.78 & July 172001 & FG004 \\
\hline Pluteus cervinus & on alder & $12 \mathrm{~V}$ & 533081.88 & 6552783.92 & July 172001 & FG005 \\
\hline Polyporus brumalis & on willow & $12 \mathrm{~V}$ & 535656.32 & 6554192.38 & July 192001 & FG023 \\
\hline Polyporus elegans & on birch or alder & $12 \mathrm{~V}$ & 535131.81 & 6553858.37 & July 182001 & FG009 \\
\hline Polyporus elegans & on wood & $12 \mathrm{~V}$ & 535131.81 & 6553858.37 & July 182001 & FG011 \\
\hline Polyporus elegans & on alder & $12 \mathrm{~V}$ & 529525 & 6553460 & July 232001 & FG055 \\
\hline Polyporus sp. & on sand & $12 \mathrm{~V}$ & 535483.64 & 6554269.03 & July 202001 & FG034 \\
\hline Russula cf. maculata & on ground & $12 \mathrm{~V}$ & 535656.32 & 6554192.38 & July 192001 & FG025 \\
\hline Russula emetica & $\begin{array}{l}\text { on sphagnum in black spruce } \\
\text { bog }\end{array}$ & $12 \mathrm{~V}$ & 537185 & 6555035 & July 222001 & FG048 \\
\hline Thelephora terrestris & on moss & $12 \mathrm{~V}$ & 532885.51 & 6552112.57 & July 172001 & FG003 \\
\hline Trichaptum biforme & on birch & $12 \mathrm{~V}$ & 532787.66 & 6552523.92 & July 172001 & FG007 \\
\hline Trichaptum biforme & on balsam poplar & $12 \mathrm{~V}$ & 509615 & 6520911 & July 242001 & FG067 \\
\hline
\end{tabular}




\section{Appendix 4. Annotated species list for Colin-Cornwall Lakes Wildland}

\begin{tabular}{|c|c|c|c|c|c|c|}
\hline Species & Habitat & \begin{tabular}{|l|} 
NAD \\
83
\end{tabular} & Easting & Northing & Date & $\begin{array}{l}\text { Specimen } \\
\text { No. }\end{array}$ \\
\hline Agrocybe praecox & on sand under burnt pine & $12 \mathrm{~V}$ & 546429 & 6605262 & 2 July 8,2002 & $\mathrm{CC} 074$ \\
\hline Agrocybe putaminum & on soil in open pine forest & $12 \mathrm{~V}$ & 546429 & 6605262 & 2July 8, 2002 & $\mathrm{CC} 073$ \\
\hline Chondrostereum purpureum & on birch & $12 \mathrm{~V}$ & 541742.77 & 6602023.6 & 6 July 6, 2002 & CC029 \\
\hline Collybia confusa & with moss under black spruce & $12 \mathrm{~V}$ & 551063 & 6608253 & \begin{tabular}{|l|l|} 
July 7,2002 \\
\end{tabular} & $\mathrm{CC} 085$ \\
\hline Collybia dryophila & with feather moss & $12 \mathrm{~V}$ & 541742.77 & 6602023.6 & July 6, 2002 & CC009 \\
\hline Collybia dryophila & on rotted wood & $12 \mathrm{~V}$ & 541742.77 & 6602023.6 & July 6, 2002 & CC044 \\
\hline Coprinus lagopoides & on burnt wood & $12 \mathrm{~V}$ & 541742.77 & 6602023.6 & July 6, 2002 & $\mathrm{CC} 036$ \\
\hline Coriolopsis gallica & on fire killed pine & $12 \mathrm{~V}$ & 541366.00 & 6599947.00 & July 7, 2002 & $\mathrm{CC} 056$ \\
\hline Coriolopsis gallica & on dead conifer & $12 \mathrm{~V}$ & 541366.00 & 6599947.00 & July 7,2002 & CC061 \\
\hline Coriolopsis gallica & on dead pine & $12 \mathrm{~V}$ & 551063 & 6608253 & 3 July 8, 2002 & $\mathrm{CC} 078$ \\
\hline Cortinarius obtusus & with moss under black spruce & $12 \mathrm{~V}$ & 551063 & 6608253 & 3 July 7, 2002 & $\mathrm{CC} 084$ \\
\hline Cortinarius obtusus & with moss under black spruce & $12 \mathrm{~V}$ & 546605.00 & 6599986.00 & July 9, 2002 & CC099 \\
\hline Fomes fomentarius & on birch & $12 \mathrm{~V}$ & 541742.77 & 6602023.6 & July 6, 2002 & $\mathrm{CC} 013$ \\
\hline Fomes fomentarius & on black spruce & $12 \mathrm{~V}$ & 541646.42 & 6602125.3 & \begin{tabular}{|l|l|} 
July 9, 2002 \\
\end{tabular} & $\mathrm{CC} 103$ \\
\hline Fomitopsis cajanderi & on fire killed pine & $12 \mathrm{~V}$ & 541366.00 & 6599947.00 & July 7,2002 & $\mathrm{CC} 054$ \\
\hline Fomitopsis cajanderi & on dead wood & $12 \mathrm{~V}$ & 541366.00 & 6599947.00 & July 7, 2002 & CC059 \\
\hline Fomitopsis pinicola & on dead conifer & $12 \mathrm{~V}$ & 541646.42 & 6602125.3 & 3 July 6, 2002 & $\mathrm{CC} 003$ \\
\hline Fomitopsis pinicola & on dead conifer & $12 \mathrm{~V}$ & 541646.42 & 6602125.3 & 3 July 6, 2002 & $\mathrm{CC} 005$ \\
\hline Fomitopsis pinicola & on dead white spruce & $12 \mathrm{~V}$ & 541742.77 & 6602023.6 & July 6, 2002 & $\mathrm{CC} 010$ \\
\hline Fomitopsis pinicola & on birch & $12 \mathrm{~V}$ & 550734.03 & 6601468.7 & 7 July 9, 2002 & $\mathrm{CC} 100$ \\
\hline Fomitopsis rosea & on rotted $\log$ & $12 \mathrm{~V}$ & 541742.77 & 6602023.6 & July 6, 2002 & $\mathrm{CC} 030$ \\
\hline Fomitopsis rosea & on cut pine stump & $12 \mathrm{~V}$ & 546429 & 6605262 & July 8, 2002 & $\mathrm{CC} 075$ \\
\hline Galerina cinctula & with moss under black spruce & $12 \mathrm{~V}$ & 551063 & 6608253 & 3 July 8, 2002 & CC081 \\
\hline Galerina mniophila & with moss under black spruce & $12 \mathrm{~V}$ & 546605.00 & 6599986.00 & July 9, 2002 & $\mathrm{CC} 097$ \\
\hline Gandoderma applanatum & on birch & $12 \mathrm{~V}$ & 541742.77 & 6602023.6 & 6 July 6, 2002 & $\mathrm{CC} 035$ \\
\hline Gleophyllum protractum & on fire killed pine & $12 \mathrm{~V}$ & 541366.00 & 6599947.00 & July 7, 2002 & $\mathrm{CC} 057$ \\
\hline Gleophyllum sepiarium & on fire killed pine & $12 \mathrm{~V}$ & 541366.00 & 6599947.00 & July 7, 2002 & $\mathrm{CC} 058$ \\
\hline Gleophyllum sepiarium & on fire killed conifer & $12 \mathrm{~V}$ & 540362.00 & 6600468.00 & July 7, 2002 & $\mathrm{CC} 066$ \\
\hline Gleophyllum sepiarium & on dead conifer & $12 \mathrm{~V}$ & 541742.77 & 6602023.6 & July 6, 2002 & $\mathrm{CC008}$ \\
\hline Gleophyllum trabium & on fire killed black spruce & $12 \mathrm{~V}$ & 543755.00 & 6602888.00 & July 8, 2002 & $\mathrm{CC} 072$ \\
\hline Gleoporus taxicola & on unpeeled pine logs of cabin & $12 \mathrm{~V}$ & 546610 & 6600000.6 & July 10,2002 & CC105 \\
\hline Gyromitra esculenta & on wood on ground & $12 \mathrm{~V}$ & 541742.77 & 6602023.6 & \begin{tabular}{|l|l|} 
July 6,2002 \\
\end{tabular} & $\mathrm{CC} 032$ \\
\hline Gyromitra esculenta & on burnt soil & $12 \mathrm{~V}$ & 540362.00 & 6600468.00 & July 7,2002 & CC064 \\
\hline Gyromitra esculenta & $\begin{array}{l}\text { on buried wood in burnt pine } \\
\text { forest }\end{array}$ & $12 \mathrm{~V}$ & 541742.77 & 6602023.6 & July 6, 2002 & CC108 \\
\hline Hebeloma cf. longicaudum & with moss under black spruce & $12 \mathrm{~V}$ & 546605.00 & 6599986.00 & July 9, 2002 & $\mathrm{CC} 096$ \\
\hline Hygrophorus sp. & $\begin{array}{l}\text { with moss under black spruce } \\
\text { (specimen missing) }\end{array}$ & $12 \mathrm{~V}$ & 551063 & 6608253 & 3 July 8,2002 & CC086 \\
\hline Inocybe lacera & on ground in burnt pine forest & $12 \mathrm{~V}$ & 541742.77 & 6602023.6 & July 6, 2002 & $\mathrm{CC} 014$ \\
\hline Inonotus obliquus & on birch & $12 \mathrm{~V}$ & 541742.77 & 6602023.6 & July 6, 2002 & $\mathrm{CC} 017$ \\
\hline Irpex lacteus & on fire killed birch & $12 \mathrm{~V}$ & 541742.77 & 6602023.6 & July 6, 2002 & $\mathrm{CC} 023$ \\
\hline Irpex lacteus & on dead wood & $12 \mathrm{~V}$ & 541742.77 & 6602023.6 & July 6, 2002 & $\mathrm{CC} 037$ \\
\hline Kuehneromyces lignicola & on burnt ground with moss & $12 \mathrm{~V}$ & 541366.00 & 6599947.00 & July 7, 2002 & $\mathrm{CC} 062$ \\
\hline Kuehneromyces lignicola & on sand under burnt pine & $12 \mathrm{~V}$ & 546429 & 6605262 & July 8, 2002 & CC111 \\
\hline Lycogala epidendrum & on burnt black spruce & $12 \mathrm{~V}$ & 543755.00 & 6602888.00 & July 8, 2002 & $\mathrm{CC} 070 \mathrm{a}$ \\
\hline
\end{tabular}




\begin{tabular}{|c|c|c|c|c|c|c|}
\hline Species & Habitat & $\begin{array}{l}\text { NAD } \\
83\end{array}$ & Easting & Northing & Date & $\begin{array}{l}\text { Specimen } \\
\text { No. }\end{array}$ \\
\hline Marasmius scorodonius & on burnt soil with moss & $12 \mathrm{~V}$ & 541742.77 & 6602023.6 & July 6, 2002 & CC04I \\
\hline Morchella elata & $\begin{array}{l}\text { on burnt ground, under white } \\
\text { spruce }\end{array}$ & $12 \mathrm{~V}$ & 541742.77 & 6602023.6 & July 6, 2002 & $\mathrm{CC} 027$ \\
\hline Mycena cf. galopus & with moss under black spruce & $12 \mathrm{~V}$ & 551063 & 6608253 & July 8, 2002 & $\mathrm{CC} 080$ \\
\hline Mycena filopes & with moss under black spruce & $12 \mathrm{~V}$ & 541366.00 & 6599947.00 & July 7, 2002 & $\mathrm{CC} 052$ \\
\hline Mycena leucogala & on burnt soil & $12 \mathrm{~V}$ & 541742.77 & 6602023.6 & July 6,2002 & $\mathrm{CC} 042$ \\
\hline $\begin{array}{l}\text { Myriosclerotinia caricic- } \\
\text { ampullaceae }\end{array}$ & on Carex sp. & $12 \mathrm{~V}$ & 541366.00 & 6599947.00 & July 7, 2002 & $\mathrm{CC} 053$ \\
\hline Neobulgaria pura & on burnt black spruce & $12 \mathrm{~V}$ & 543755.00 & 6602888.00 & July 8, 2002 & $\mathrm{CC} 070 \mathrm{~b}$ \\
\hline Omphalina ericetorum & with moss under black spruce & $12 \mathrm{~V}$ & 541742.77 & 6602023.6 & July 6,2002 & $\mathrm{CC} 046$ \\
\hline Omphalina ericetorum & with moss under black spruce & $12 \mathrm{~V}$ & 551063 & 6608253 & July 8, 2002 & $\mathrm{CC} 082$ \\
\hline Peziza praetervisa & on burnt soil & $12 \mathrm{~V}$ & 541742.77 & 6602023.6 & July 6,2002 & $\mathrm{CC} 011$ \\
\hline Phellinus chrysoloma & on fire killed pine & $12 \mathrm{~V}$ & 541366.00 & 6599947.00 & July 7, 2002 & $\mathrm{CC} 055$ \\
\hline Phellinus chrysoloma & on dead white spruce & $12 \mathrm{~V}$ & 541366.00 & 6599947.00 & July 7, 2002 & $\mathrm{CC} 063$ \\
\hline Phellinus chrysoloma & on unpeeled pine logs of cabin & $12 \mathrm{~V}$ & 546610 & 6600000.6 & July 10, 2002 & \begin{tabular}{|l|l|} 
CC104 \\
\end{tabular} \\
\hline Phellinus ignarius & on dead wood & $12 \mathrm{~V}$ & 541742.77 & 6602023.6 & July 6,2002 & CC048 \\
\hline Phellinus ignarius & on birch & $12 \mathrm{~V}$ & 541366.00 & 6599947.00 & July 7, 2002 & CC049 \\
\hline Phellinus igniarius & on birch & $12 \mathrm{~V}$ & 541742.77 & 6602023.6 & July 6,2002 & $\mathrm{CC} 033$ \\
\hline Phellinus igniarius & on birch & $12 \mathrm{~V}$ & 541742.77 & 6602023.6 & July 6,2002 & $\mathrm{CC} 034$ \\
\hline Phellinus igniarius & on birch & $12 \mathrm{~V}$ & 529639.00 & 6600841.00 & July 9, 2002 & CC087 \\
\hline Phellinus tremulae & on aspen popular & $12 \mathrm{~V}$ & 541742.77 & 6602023.6 & July 6,2002 & $\mathrm{CC} 015$ \\
\hline Pholiota spumosa & on soil & $12 \mathrm{~V}$ & 541742.77 & 6602023.6 & July 6,2002 & $\mathrm{CC} 021$ \\
\hline Pholiota spumosa & on soil & $12 \mathrm{~V}$ & 541742.77 & 6602023.6 & July 6, 2002 & $\mathrm{CC} 022$ \\
\hline Pholiota spumosa & $\begin{array}{l}\text { on wood in burnt ground, under } \\
\text { white spruce }\end{array}$ & $12 \mathrm{~V}$ & 541742.77 & 6602023.6 & July 6, 2002 & $\mathrm{CC} 026$ \\
\hline Pholiota spumosa & $\begin{array}{l}\text { on wood in burnt ground, under } \\
\text { white spruce }\end{array}$ & $12 \mathrm{~V}$ & 541742.77 & 6602023.6 & July 6, 2002 & $\mathrm{CC} 028$ \\
\hline Pholiota spumosa & on burnt soil & $12 \mathrm{~V}$ & 540362.00 & 6600468.00 & July 7, 2002 & $\mathrm{CC} 065$ \\
\hline Pholiota spumosa & on burnt soil with moss & $12 \mathrm{~V}$ & 543755.00 & 6602888.00 & July 8, 2002 & CC071 \\
\hline Pholiota spumosa & on buried wood & $12 \mathrm{~V}$ & 551063 & 6608253 & July 8, 2002 & $\mathrm{CC} 077$ \\
\hline Piptoporus betulinus & on birch & $12 \mathrm{~V}$ & 541742.77 & 6602023.6 & July 6,2002 & $\mathrm{CC} 020$ \\
\hline Polyporus elegans & on dead wood & $12 \mathrm{~V}$ & 541742.77 & 6602023.6 & July 6,2002 & $\mathrm{CC} 047$ \\
\hline Polyporus elegans & on birch & $12 \mathrm{~V}$ & 541366.00 & 6599947.00 & July 7,2002 & $\mathrm{CC} 050$ \\
\hline Polyporus elegans & on birch & $12 \mathrm{~V}$ & 529639.00 & 6600841.00 & July 9, 2002 & $\mathrm{CC} 088$ \\
\hline Polyporus melanopus & on rotted $\log$ & $12 \mathrm{~V}$ & 541742.77 & 6602023.6 & July 6,2002 & $\mathrm{CC} 019$ \\
\hline Polyporus varius & on birch & $12 \mathrm{~V}$ & 529639.00 & 6600841.00 & July 9, 2002 & $\mathrm{CC} 093$ \\
\hline Polyporus varius & on birch & $12 \mathrm{~V}$ & 546610 & 6600000.6 & July 10, 2002 & CC106 \\
\hline Psathyrella cf. phegophila & $\begin{array}{l}\text { on burnt ground, under white } \\
\text { spruce }\end{array}$ & $12 \mathrm{~V}$ & 541742.77 & 6602023.6 & July 6, 2002 & $\mathrm{CC} 025$ \\
\hline Pycnoporus cinnabarinus & on birch & $12 \mathrm{~V}$ & 540362.00 & 6600468.00 & July 7,2002 & CC068 \\
\hline Sarcodon scabrosus & on ground & $12 \mathrm{~V}$ & 541646.42 & 6602125.3 & July 6,2002 & CC006 \\
\hline Skeletocutis amorpha & on alder & $12 \mathrm{~V}$ & 541742.77 & 6602023.6 & July 6, 2002 & $\mathrm{CC} 018$ \\
\hline Skeletocutis amorpha & on birch & $12 \mathrm{~V}$ & 529639.00 & 6600841.00 & July 9, 2002 & $\mathrm{CC} 092$ \\
\hline Skeletocutis amorpha & on burnt alder & $12 \mathrm{~V}$ & 541646.42 & 6602125.3 & July 9, 2002 & $\mathrm{CC} 101$ \\
\hline Specimen Missing & burnt soil & $12 \mathrm{~V}$ & 541742.77 & 6602023.6 & July 6,2002 & CC038 \\
\hline Specimen Unidentifiable & on dead spruce & $12 \mathrm{~V}$ & 541742.77 & 6602023.6 & July 6,2002 & $\mathrm{CC} 031$ \\
\hline Stemonitis splendens & on birch & $12 \mathrm{~V}$ & 529639.00 & 6600841.00 & July 9, 2002 & CC091 \\
\hline Stereum hirsutum & on birch & $12 \mathrm{~V}$ & 540362.00 & 6600468.00 & July 7, 2002 & $\mathrm{CC} 067$ \\
\hline
\end{tabular}




\begin{tabular}{|c|c|c|c|c|c|c|}
\hline Species & Habitat & $\begin{array}{l}\text { NAD } \\
83\end{array}$ & Easting & Northing & Date & $\begin{array}{l}\text { Specimen } \\
\text { No. }\end{array}$ \\
\hline Stereum striatum & on birch & $12 \mathrm{~V}$ & 541366.00 & 6599947.00 & July 7, 2002 & $\mathrm{CC} 060$ \\
\hline Thelephora terrestris & with moss under black spruce & $12 \mathrm{~V}$ & 551063 & 6608253 & July 8, 2002 & $\mathrm{CC} 083$ \\
\hline Trametes cervina & $\log$ in cabin wall & $12 \mathrm{~V}$ & 541742.77 & 6602023.6 & July 6, 2002 & $\mathrm{CC} 016$ \\
\hline Trametes hirsuta & on dead black spruce & $12 \mathrm{~V}$ & 546605.00 & 6599986.00 & July 9, 2002 & $\mathrm{CC} 095$ \\
\hline Trametes hirsuta & on fire killed pine & $12 \mathrm{~V}$ & 541366.00 & 6599947.00 & July 7, 2002 & $\mathrm{CC} 110$ \\
\hline Trametes suaveolens & on birch & $12 \mathrm{~V}$ & 541646.42 & 6602125.3 & July 9, 2002 & $\mathrm{CC} 102$ \\
\hline Trametes versicolor & on dead pine & $12 \mathrm{~V}$ & 546429 & 6605262 & July 8, 2002 & $\mathrm{CC} 076$ \\
\hline Trichaptum abietinum & on white spruce & $12 \mathrm{~V}$ & 529639.00 & 6600841.00 & July 9, 2002 & $\mathrm{CC} 090$ \\
\hline Trichaptum biforme & on fire killed birch & $12 \mathrm{~V}$ & 541646.42 & 6602125.3 & July 6, 2002 & $\mathrm{CCO01}$ \\
\hline Trichaptum biforme & on birch & $12 \mathrm{~V}$ & 541646.42 & 6602125.3 & July 6, 2002 & $\mathrm{CCOO2}$ \\
\hline Trichaptum biforme & on dead wood & $12 \mathrm{~V}$ & 541742.77 & 6602023.6 & July 6, 2002 & $\mathrm{CC007}$ \\
\hline Trichaptum biforme & on birch & $12 \mathrm{~V}$ & 541742.77 & 6602023.6 & July 6, 2002 & $\mathrm{CC} 012$ \\
\hline Trichaptum biforme & on fire killed birch & $12 \mathrm{~V}$ & 541742.77 & 6602023.6 & July 6, 2002 & $\mathrm{CCO} 24$ \\
\hline Trichaptum biforme & on birch & $12 \mathrm{~V}$ & 541742.77 & 6602023.6 & July 6, 2002 & $\mathrm{CC} 045$ \\
\hline Trichaptum biforme & on fire killed birch & $12 \mathrm{~V}$ & 540362.00 & 6600468.00 & July 7, 2002 & $\mathrm{CC069}$ \\
\hline Trichaptum biforme & on birch & $12 \mathrm{~V}$ & 529639.00 & 6600841.00 & July 9, 2002 & CC094 \\
\hline Tubaria hiemalis & balsam poplar on the ground & $12 \mathrm{~V}$ & 541646.42 & 6602125.3 & July 6, 2002 & $\mathrm{CCO04}$ \\
\hline Tubaria hiemalis & on wood & $12 \mathrm{~V}$ & 541366.00 & 6599947.00 & July 7, 2002 & $\mathrm{CC} 051$ \\
\hline Tubaria romagnesiana & on rotted birch & $12 \mathrm{~V}$ & 541742.77 & 6602023.6 & July 6, 2002 & CC039 \\
\hline Tubaria romagnesiana & on burnt soil & $12 \mathrm{~V}$ & 541742.77 & 6602023.6 & July 6, 2002 & $\mathrm{CC} 040$ \\
\hline Unidentifiable & on birch & $12 \mathrm{~V}$ & 529639.00 & 6600841.00 & July 9, 2002 & CC089 \\
\hline Unidentifiable yellow slime & on sphagnum & $12 \mathrm{~V}$ & 541366.00 & 6599947.00 & July 10,2002 & $2 \mathrm{CC} 109$ \\
\hline Xeromphalina fraxinophila & on burnt soil with moss & $12 \mathrm{~V}$ & 541742.77 & 6602023.6 & July 6, 2002 & $\mathrm{CC} 043$ \\
\hline Xeromphalina fraxinophila & with moss under black spruce & $12 \mathrm{~V}$ & 551063 & 6608253 & July 8, 2002 & CC079 \\
\hline Xeromphalina fraxinophila & with moss under black spruce & $12 \mathrm{~V}$ & 546605.00 & 6599986.00 & July 9, 2002 & $\mathrm{CC} 098$ \\
\hline Xeromphalina fraxinophila & with moss under black spruce & $12 \mathrm{~V}$ & 541366.00 & 6599947.00 & July 7, 2002 & $\mathrm{CC} 107$ \\
\hline
\end{tabular}




\section{Appendix 5. Partial list of literature accounts of fungi occurrences in Alberta.}

Abbott, L. L. 1990. Ministik Lake Foray. The Stinkhorn, 4 (1):17. Edmonton.

Abbott, L. L. 1991. Ministik Lake Foray 1990. The Stinkhorn, 5 (1):11. Edmonton.

Abbott, L. L. 1991. Shaw Lake Foray 1990. The Stinkhorn, 5 (1):20-21. Edmonton.

Abbott, S. P. 1987. A Return Foray. The Stinkhorn, 1 (1):12. Edmonton.

Abbott, S. P. 1987. EMS Display at the Provincial Museum. The Stinkhorn, 4 (1):29-31. Edmonton.

Abbott, S. P. 1987. EMS Mushroom Display. The Stinkhorn, 1 (1):19-20. Edmonton.

Abbott, S. P. 1987. Foray at the Botanic Garden. The Stinkhorn, 1 (1):14-15. Edmonton.

Abbott, S. P. 1987. The First Foray. The Stinkhorn, 1 (1):13. Edmonton.

Abbott, S. P. 1989. Key to Boleti of Alberta. University of Alberta, Edmonton. 12pp. Mimeograph.

Abbott, S. P. 1990. Amanita muscaria Poisonings. The Stinkhorn, 4 (1): 21. Edmonton.

Abbott, S. P. 1990. Entoloma Grande Poisonings. The Stinkhorn, 4 (1): 20. Edmonton.

Abbott, S. P. 1990. Pet Poisonings -- A Narrative.... The Stinkhorn, 5 (1): 22. Edmonton.

Abbott, S. P. 1990. Shaw Lake. The Stinkhorn, 4 (1): 38-42. Edmonton.

Abbott, S. P. 1990. Stinkhorns. The Stinkhorn, 4 (1): 32. Edmonton.

Abbott, S. P. and L. L. Abbott. 1991. Alberta Verpa Peport. The Stinkhorn, 5 (1): 13-18. Edmonton.

Abbott, S. P. and R. S. Currah. 1989. The larger cup fungi and other Ascomycetes of Alberta, an annotated checklist. Devonian Botanic Garden, University of Alberta, Edmonton. 96 pp.

Abbott, S.P. \& R.S. Currah. 1997. The Helvellaceae: Systematic revision and occurrence in northern and northwestern North America. Mycotaxon 62:1-125.

Abbott, S.P. 1992. Systematic studies of the Helvellaceae in northern and northwestern North America. University of Alberta. (Canadian Theses Service microfiche TH 77275 National Library)

Abbott, S.P. and R.S. Currah. 1997. The Helvellaceae: systematic revision and occurrence in northern and northwestern North America. Mycotaxon 62: 1-125.

Abbott, S.P., and Currah. R.S. 1988. The genus Helvella in Alberta. Mycotaxon 33: 229-250.

Addy, H.D., Hambleton, S. and Currah, R.S. 2000. Distribution and molecular characterization of the root endophyte Phialocephala fortinii along an environmental gradient in the boreal forest of Alberta. Mycol. Res. 104: 1213-1221.

Anonymous 1991. Stinkhorn Notes. The Stinkhorn, 5 (1):40. Edmonton

Baranyay, J. A. 1967. Notes on Hypoxylon canker of aspen in Alberta. For. Chron. 43:372-280.

Baranyay, J.A. 1968.Fungi collected during forest diseases surveys in northern Alberta and the district of Mackenzie, Northwest Territories. For Branch Dept. Public no. 1238, 25p.

Boyetchko, S.M, and J. P. Tewari. 1993. Occurrence of vesicular-arbuscular mycorrhizal fungi in Alberta, Canada. Zeitschrift Fur Naturforschung C-A Journal of Biosciences. 48(11-12):923-929.

Bradbury, SM. 1998. Ectomycorrhizas of lodgepole pine (Pinus contorta) seedlings originating from seed in southwestern Alberta cut blacks. Can. J. Bot.- Rev. Can. Bot. 76: 213-217.

Brodie, H. J. 1966. A new species of Cyathus from the Canadian Rockies. Canadian J. Bot. 44:1235-1237.

Brodie, H. J. 1968. The Nidulariaceae of Canada. Canadian Field-Naturalist 82: 2-14 
Brodie, H.J. 1970. A previously unnamed species of Cyathus from the Cypress Hills, Alberta. Canadian Journal of Botany. 48: 749-750

Carmichael, J. W., W. B. Kendrick, I. L. Connerse and L. Sigler. 1980. Genera of Hyphomycetes. The Univ. of Alberta Press, Edmonton, Canada. 386p.

Cormack, M.W. 1948. Winter crown rot or snow mold of alfalfa, clovers, and grasses in Alberta. Can. I. Res. (Sec. C) $26: 71-85$.

Currah RS; Smreciu EA; Lehesvirta T; Niemi M; Larsen KW. 2000. Fungi in the winter diets of northern flying squirrels and red squirrels in the boreal mixedwood forest of northeastern Alberta. Canadian Journal of Botany-Revue Canadienne de Botanique. 78(12):1514-1520.

Currah, R. 1986. A new species of Ascodesmis from Alberta. Mycologia 78: 198-201.

Danielson R.M. 1984. Ectomycorrhizal associations in jack pine stands in northeastern Alberta. Canadian Journal of Botany 62: 932-939.

Danielson, R. M. (no date). Key to Mushrooms and their allies found in Alberta. University of Calgary. Mimeo. $66 \mathrm{p}$.

Danielson, R.M. 1979. Hypogeous ascomycetes in Alberta Canada, with two new North American records. Mycotaxon, 9:445-450

Danielson, R.M., and S. Visser. 1989. Host response to innoculation and behaviour of introduced and indigenous ectomycorrhizal fungi of jack pine grown on oil-sands tailings. Canadian Journal of Forest Research vol. 19: $1412-1421$

Danielson, R.M.1984. Ectomycorrhizal associations in jackpine stands in northeastern Alberta, Can. J. Bot. 62:932-939.

Gilbertson, R. L. and L. Ryvarden. 1986. North American Polypores Vol. 1. Fungiflora, Oslo, Norway. 433p.

Gilbertson, R. L. and L. Ryvarden. 1987. North American Polypores Vol. II. Fungiflora, Oslo, Norway. 885p.

Gilbertson, R.L. 1981. North American wood-rotting fungi that cause brown rots. Mycotaxon 12:372-416.

Ginns, J. 1989. Descriptions and notes for some unusual North American corticioid fungi (Aphyllophorales, Corticiaceae). Memoirs N.Y. Botanical Garden 49: 129-137.

Hambleton, S., Huhtinen, and S. Currah, R.S. 1999. Hymenoscyphus ericae: a new record from western Canada. Mycol. Res. 103:1391-1397.

Hiratsuka, Y., A.A. Loman. 1984.Decay of aspen and balsam poplar in Alberta. Can. For. Res, Cent. North. For. Res. Cent. Edmonton Alberta. Inf. Rep. NOR-X-262.

Hiratsuka, Y., Gibbard, D.A., Bakowsky, O., and Maier, G.B. 1990. Classification and measurement of aspen decay and stain in Alberta. For. Can. Northwest Reg., North. For. Cent., Edmonton, Alberta. Inf. Rep. NOR$\mathrm{X}-314.29 \mathrm{p}$.

Hiratsuka, Y., P. J. Maruyama. 1985. Western gall rusts. Can. For. Res, Cent. North. For. Res. Cent. Edmonton Alberta. Pest Leafl. NFRC PL 27-85.

Hutchison, L.J. 1999. Wood-inhabiting microfungi isolated from Populus tremuloides from Alberta and northeastern British Columbia. Can. J. Bot. 77: 898-905.

Hutchison, L.J. 1999. Wood-inhabiting microfungi isolated from Populus tremuloides from Alberta and northeastern Brotidh Columbia. Can. J. Bot. 77: 898-905.

Kernaghan, G. and Currah, R.S. 1998. Ectomycorrhizal fungi at tree line in the Canadian Rockies. Mycotaxon 69: 39-80.

Kernaghan, G., Currah, R.S. and Bayer, R.J. 1997. Russulaceous ectomycorrhizae of Abies lasiocarpa and Picea engelmannii. Canadian Journal of Botany 75: 1843-1850. 
Larsen, H. J. and W. C. Denison. 1978. A checklist of operculate cup-fungi (Pezizales) of North America west of the Great Plains. Mycotaxon 7:68-90.

Lawrence J.J. and Hiratsuka. 1972. Forest fungi collection in Banff National Park, Alberta. Information Report NOR-X-22, Northern Forest Research Centre.

Lawrence J.J. and Hiratsuka. 1972. Forest fungi collection in Jasper National Park, Alberta. Information Report NOR-X-21, Northern Forest Research Centre.

Lawrence J.J. and Hiratsuka. 1972. Forest fungi collection in Waterton Lakes National Park, Alberta. Information Report NOR-X-20, Northern Forest Research Centre.

Loman, A. A., G. Paul. 1963. Decay of lodgepole pine in two foothills sections of the boreal forest in Alberta. For Chron. 39:422-435

Lowe J.L. \& R.L. Gilbertson. 1961, Synopsis of Polyporaceae of the Western United States and Canada. Mycoloia vol. 53.

Lumley, T.C., S.P. Abbott \& R.C. Currah. 2000. Microscopic ascomycetes isolated from rotting wood in the boreal forest. Mycotaxon 74:395-414.

Marcia Monreal, S.M. Berch, and Mary Berbee. 1999. Molecular diversity of ericoid mycorrhizal fungi. Can. J. Bot. 77: 1580-1594.

Martin K.J. \& R. L. Gilbertson 1977. Synopsis of wood-rotting fungi on spruce in North America. Mycotaxon 6: 43-77.

Mugala, M.S., P.V. Blenis, Y. Hiratsuka, and K.I. Mallet. 1989. Infection of lodgepole pine and white spruce by Alberta isolates of Armillaria. Canad. J. For. Res. 19: 685-689.

Parmelee, J. A. 1971. The genus Gymnosporangium in western Canada. Can. J. Bot. 49:903-926

Parmelee, J.A. 1984. Microfungi parasitic on vascular plants in Waterton Lakes National Park, Alberta, and environs. Agriculture Canada Tech. Bull. 1984-11E, Ottawa. 32 pp.

Paul, G. and Etheridge, D.E. 1958. Decay of aspen (Populus tremuloides Michx.) and balsam poplar (P. balsamifera L.) in the Lesser Slave Lake region of Alberta. Alberta Dep. Land For., For. Serv. Div., Edmonton, Alberta, and Can. Dep. Agric., For. Biol. Div., V+Calgary, Alberta, Mimeograph.

Redhead, S.A. 1984. Arrhenia and Rimbachia, expanded generic concepts, and a reevaluation of Leptoglossum with emphasis on muscicolous North American taxa. Canad. J. Bot. 62: 865-892.

Redhead, S.A. 1989. A biogeographical overview of the Canadian mushroom flora. Canad. J. Bot. 67: 3003-3062.

Robinson-Jeffrey. C., A. A. Loman. 1963. Fungi Isolated in culture from red heartwood stain and advanced decay of lodgepole pine in Alberta. Can. J. Bot. 41:1371-1375.

Schalkwijk, H.M.E. 1975. Mushrooms of the Edmonton Area- edible and poisonous. Printed and published in Edmonton 34pp.

Schalkwijk, H.M.E. 1989. Leni Schalkwijk's checklist of Alberta Fungi. The Stinkhorn, Edmonton 3 (1): 1-66.

Schalkwijk-Barendsen, H.M.E. 1991. Checklist of fungi to accompany the book Mushrooms of Western Canada. Schalkwijk-Barendsen, Edmonton, Alta. published by author.

Schalkwijk-Barendsen, H.M.E. 1991. Mushrooms of western Canada. Lone Pine Publishing, Edmonton. 414 pp.

Schalkwyk, H.M.E. 1987. Notes from members. The Stinkhorn. 1 (1): 23. Edmonton

Schalkwyk, H.M.E. 1989. Leni Schalkwyk's checklist of Alberta Fungi. The Stinkhorn. 3 (1): 1-66. Edmonton

Schulz, M. 2002. Common Wood Decay Fungi And Polypores of the Edmonton Area. Mycologist, Department of Biological Science. University of Alberta. Edmonton Alberta. Mimeograph

Sigler, L. and A.L. Flis. 1998. University of Alberta microfungus collection and herbarium (UAMH) Devonian Botanic Garden, Edmonton, AB, Canada. 
Stoyke, G., and Currah, R.S. 1991. Endophytic fungi from the mycorrhizae of alpine ericoid plants. Can. J. Bot. 69: $347-352$.

Thomas, G. P. , D. E. Etheridge, G. Paul. 1960. Fungi and decay in aspen and balsam poplar in the boreal forest region, Alberta. Can J. Bot.38:549-466.

Thormann MN; Currah RS; Bayley SE. 2002. The relative ability of fungi from Sphagnum fuscum to decompose selected carbon substrates. CANADIAN JOURNAL OF MICROBIOLOGY. 48(3):204-211.

Traquair, J.A. 1980. The first Mary Elliot Mycological foray. Alberta Naturalist. 10: 4-6

Tsuneda, A., Thormann, M.N., and R.S. Currah. 2000. Scleroconidioma , a new genus of dematiaceous Hyphomycetes. Can. J. Bot. 78:1294-1298.

Zalasky, H. 1964. Nomenculture and description of Diplodia tumefaciens (Shear) Zalasky (=Macrophoma tumefaciens Shear apud Hubert). Can. J, Bot. 42:1049-1055.

Ziller, W. G. 1974. The Tree Rusts of Western Canada. Canadian Forestry Service Pub. No. 1329. Pacific Forest Research Centre, Victoria, B. C. 272p. 
Appendix 6. Photographs of macrofungi from La Butte Creek, Fidler-Greywillow and Colin-Cornwall Lakes Wildland Provincial Parks

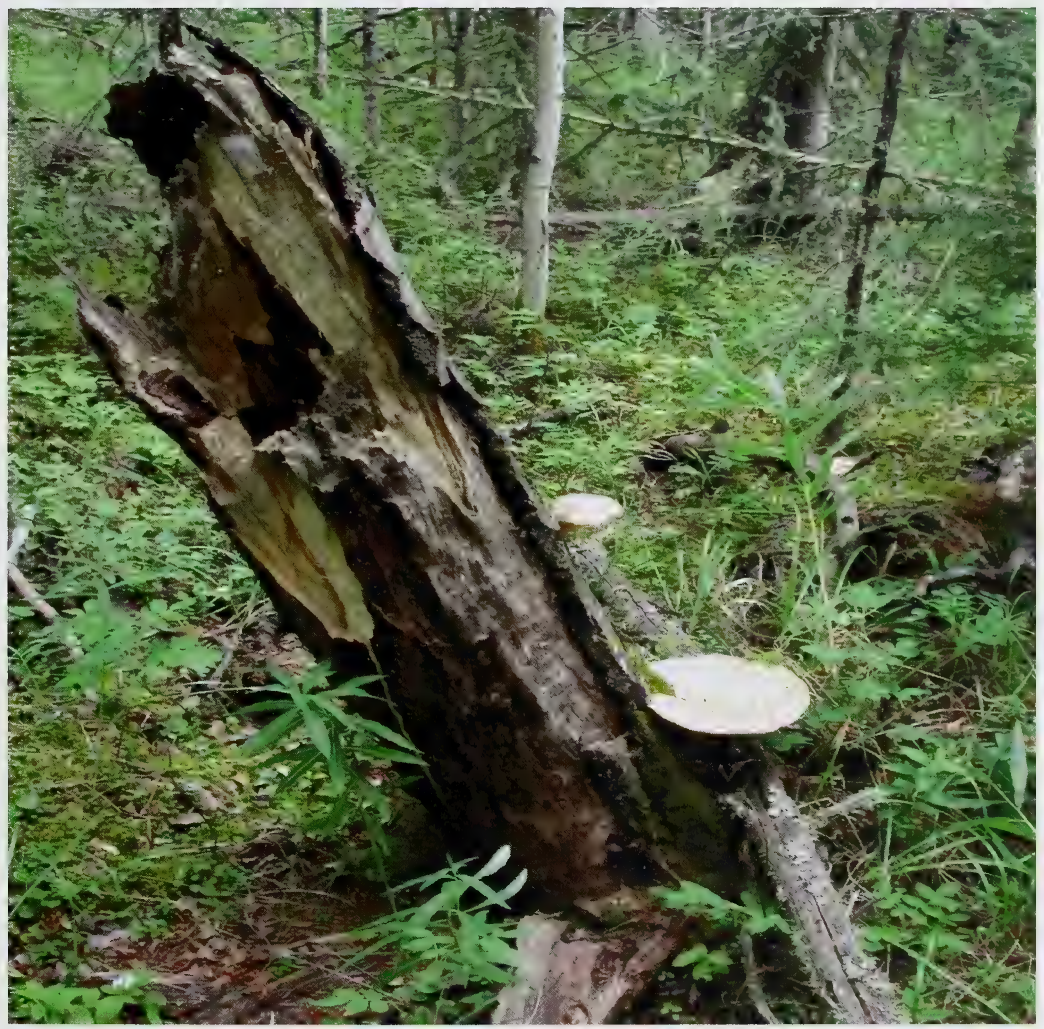

LB061 Polyporus squamosus, La Butte Creek Wildland Provincial Park. Photographer: Drajs Vujnovic 


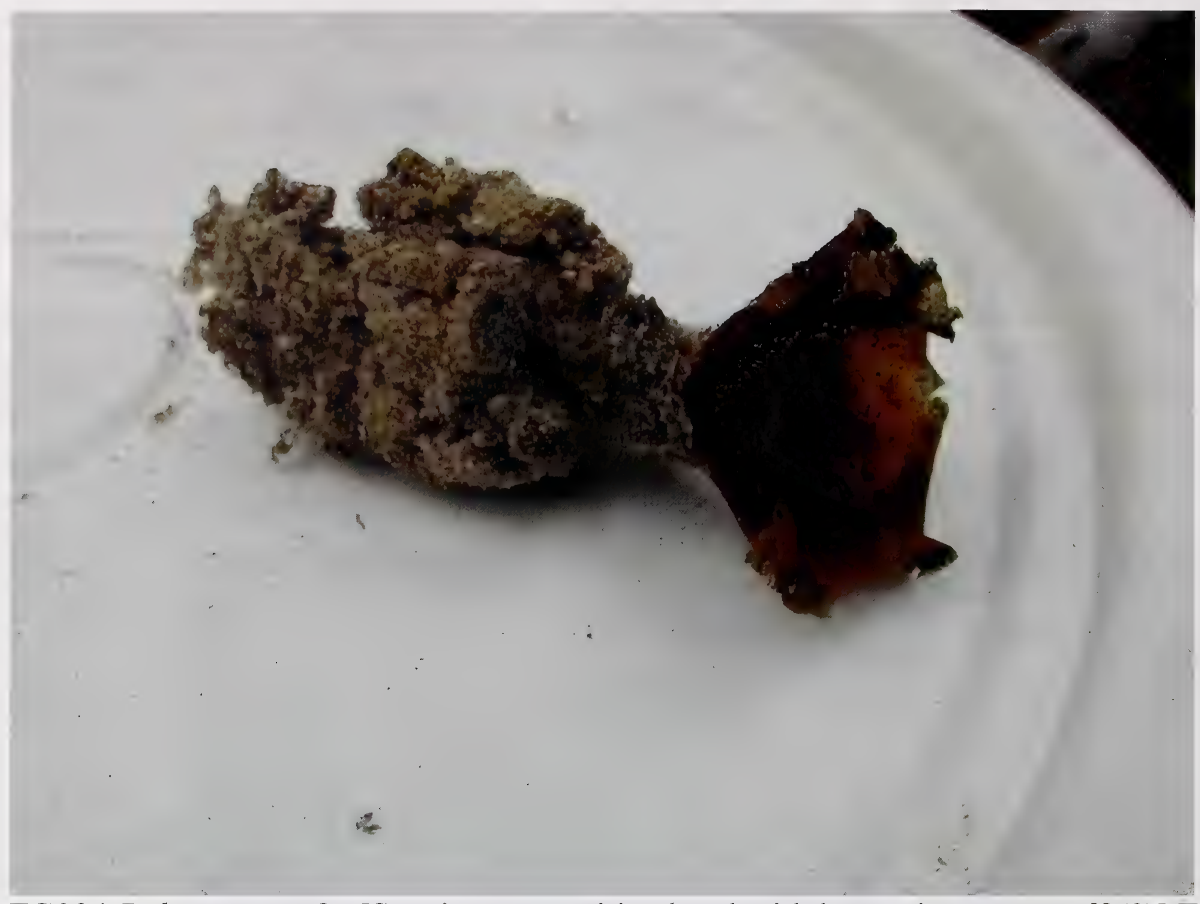

FG034 Polyporus sp.? , [Specimen parasitized and with hymenium eaten off (?)] FidlerGreywillow Wildland Provincial Park.

Photographer: Drajs Vujnovic

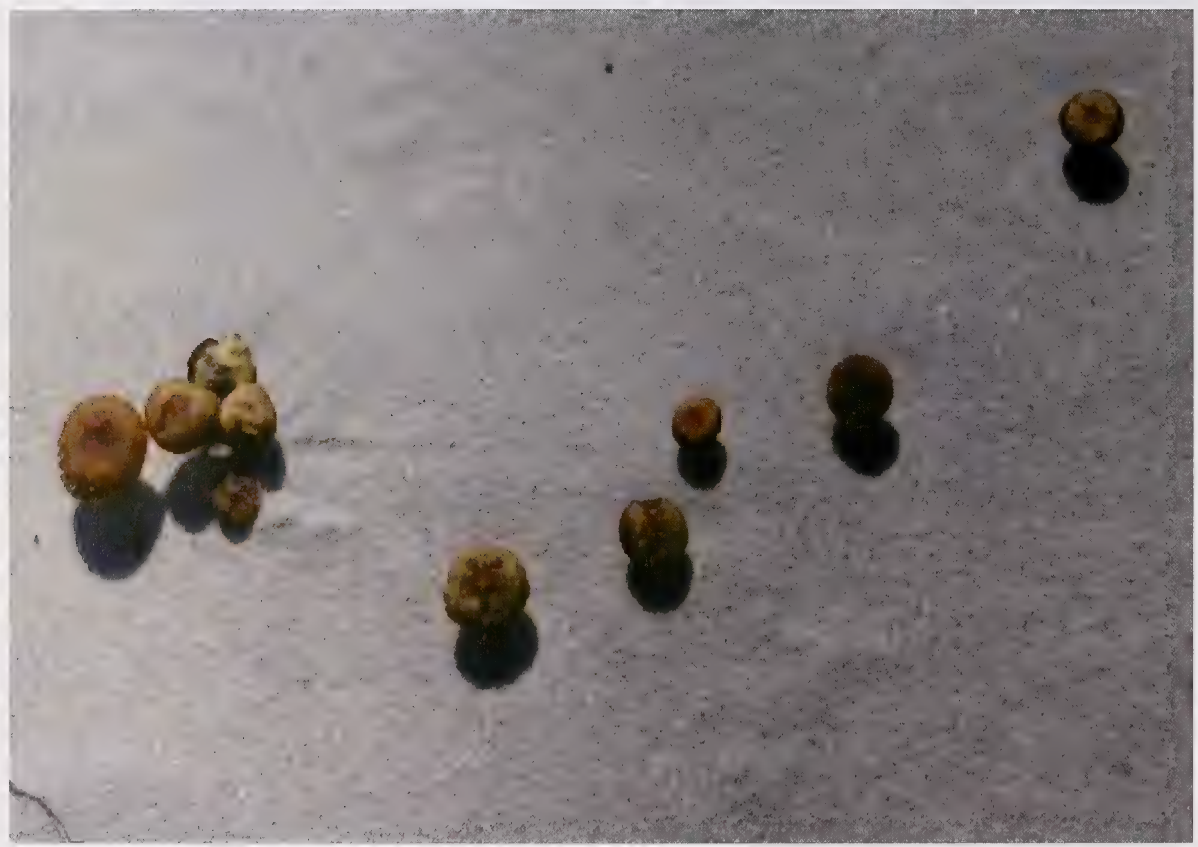

FG053 Cortinarius brunneus var. grandicolor, on sand at lake edge, Fidler-Greywillow Wildland Provincial Park.

Photographer: Lorna Allen 


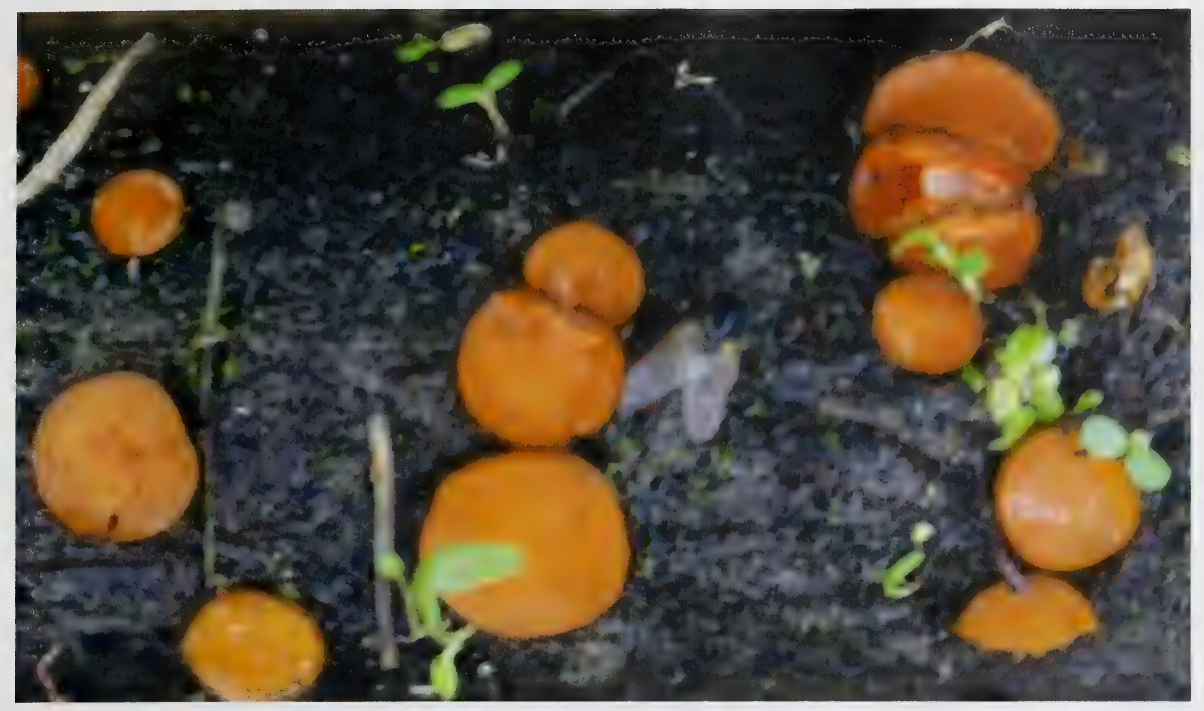

FG024 Scutellinia scutellata, on mud of beaver dam, Fidler-Greywillow Wildland Provincial Park.

Photographer: Lorna Allen

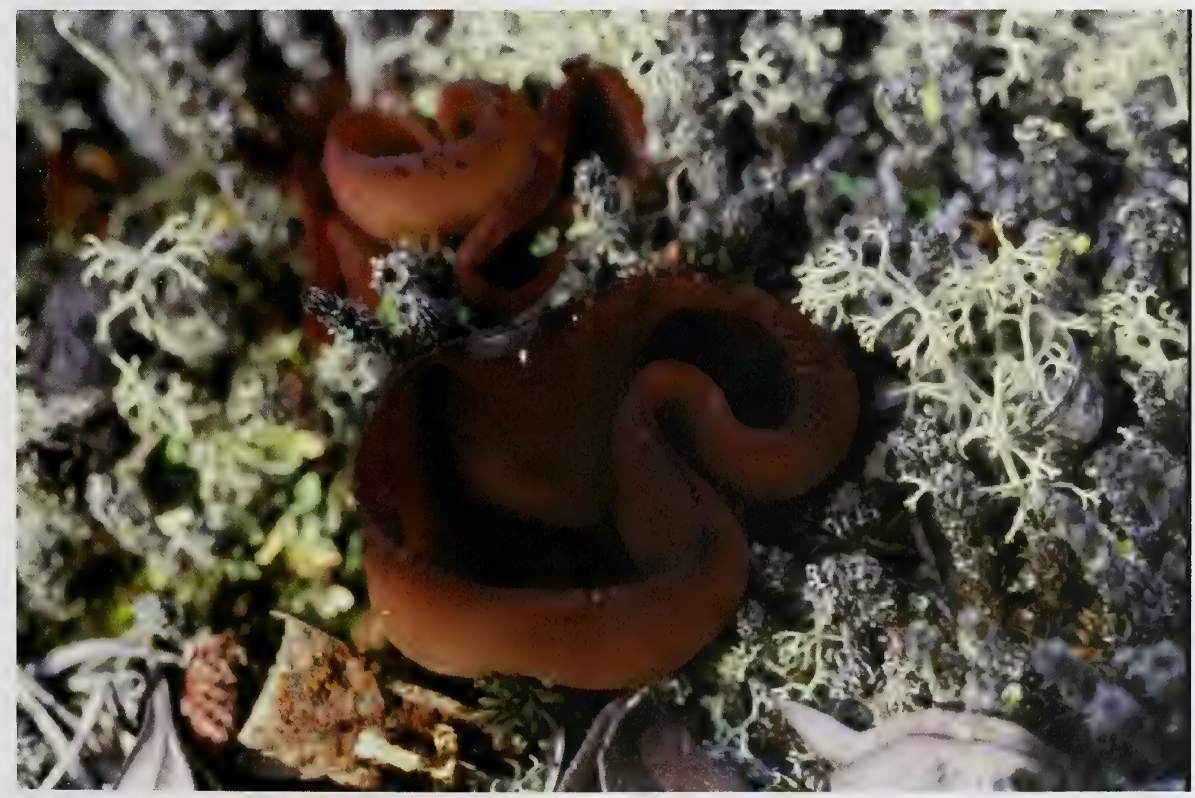

FG016 Peziza badia, Fidler-Greywillow Wildland Provincial Park.

Photographer: Lorna Allen 


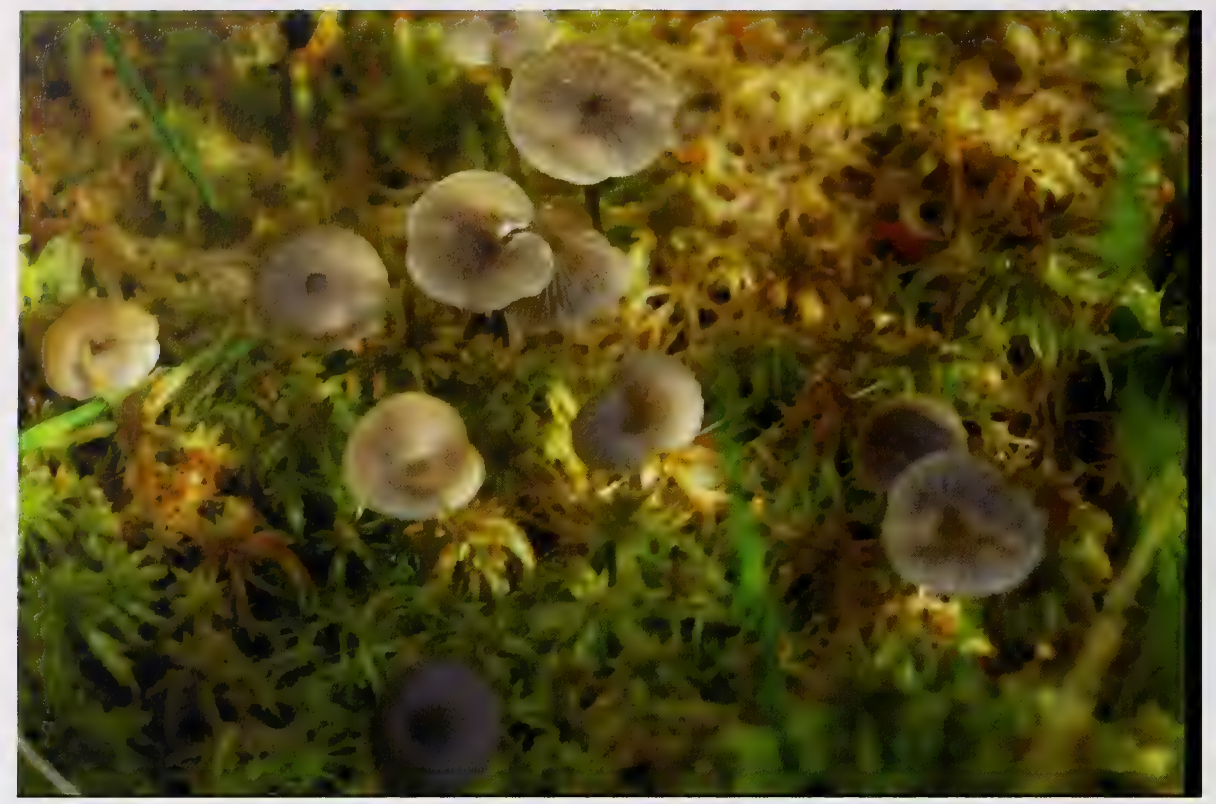

FG001 Omphalina ericetorum, Fidler-Greywillow Wildland Provincial Park. Photographer: Lorna Allen

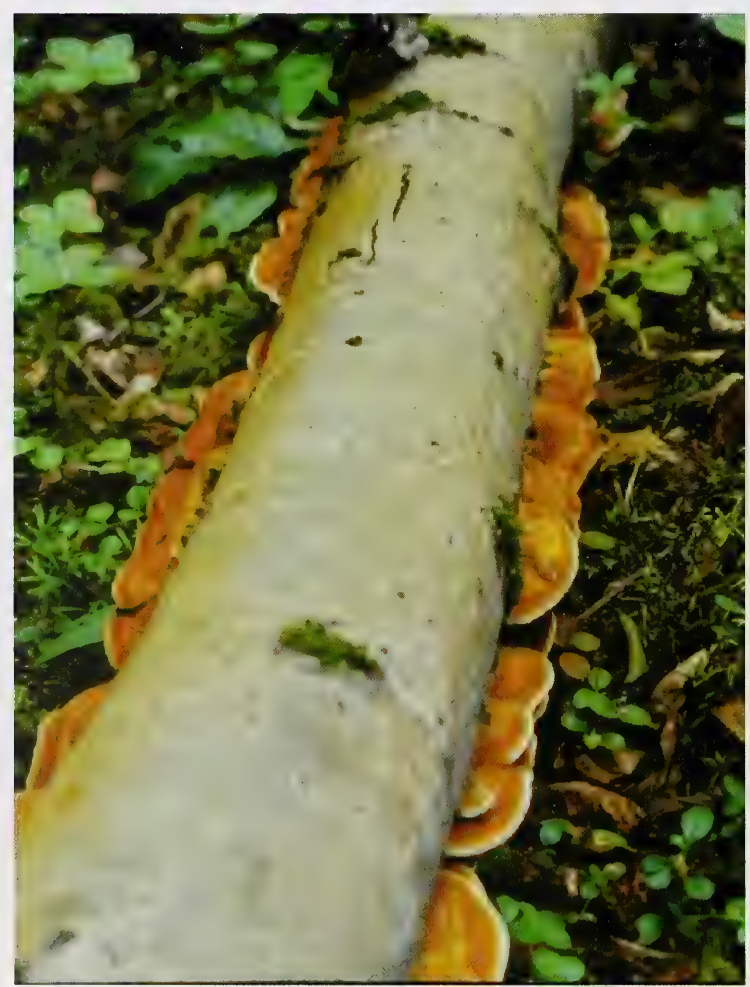

LB082 Coriolopsis gallica, La Butte Creek Wildland Provincial Park.

Photographer: Lorna Allen 


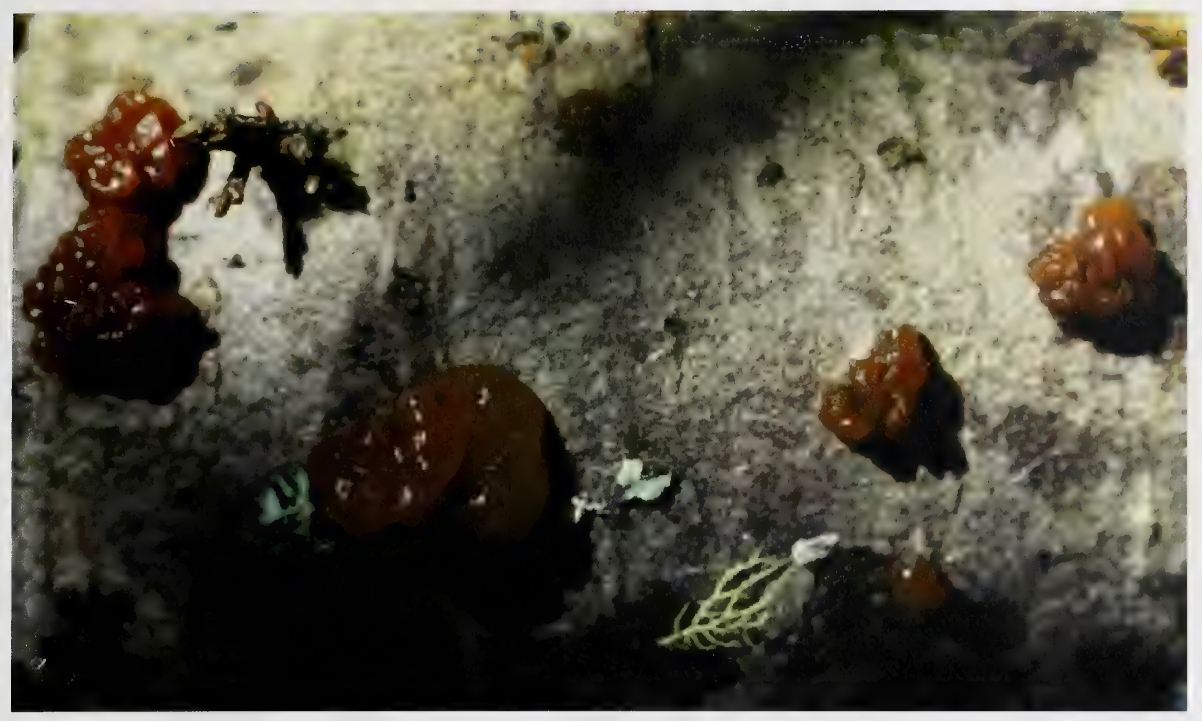

LB074 Exidea glandosa, La Butte Creek Wildland Provincial Park. Photographer: Lorna Allen

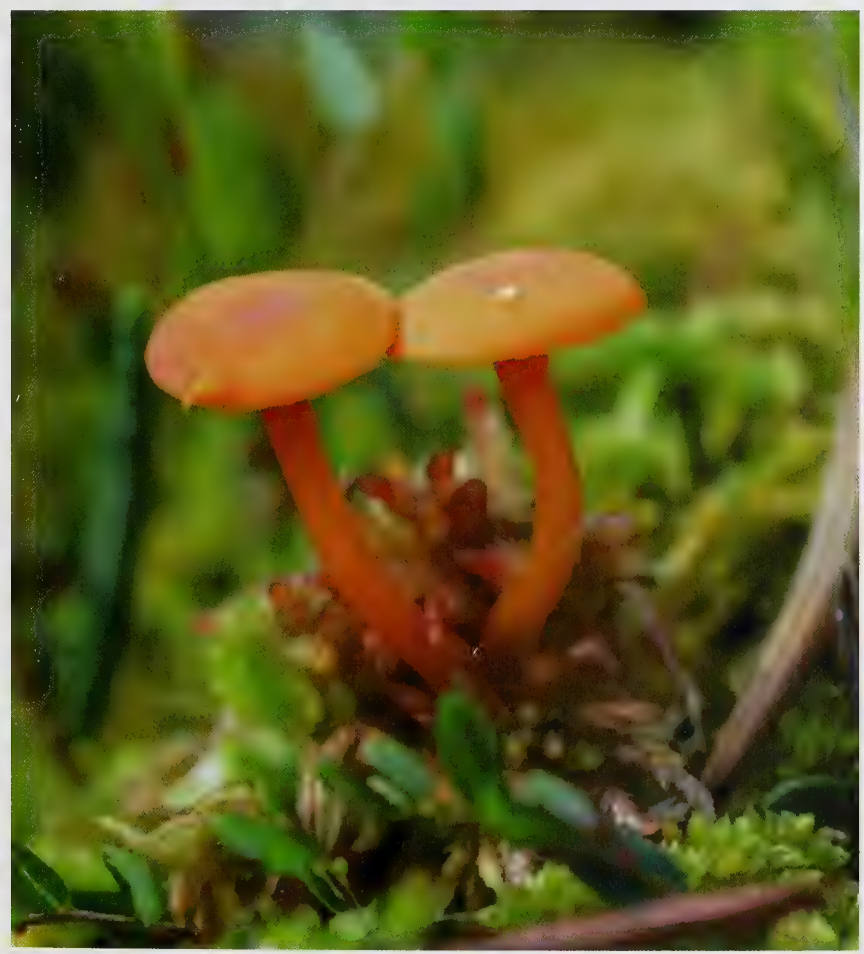

Laccaria sp., La Butte Creek Wildland Provincial Park.

Photographer: Lorna Allen 


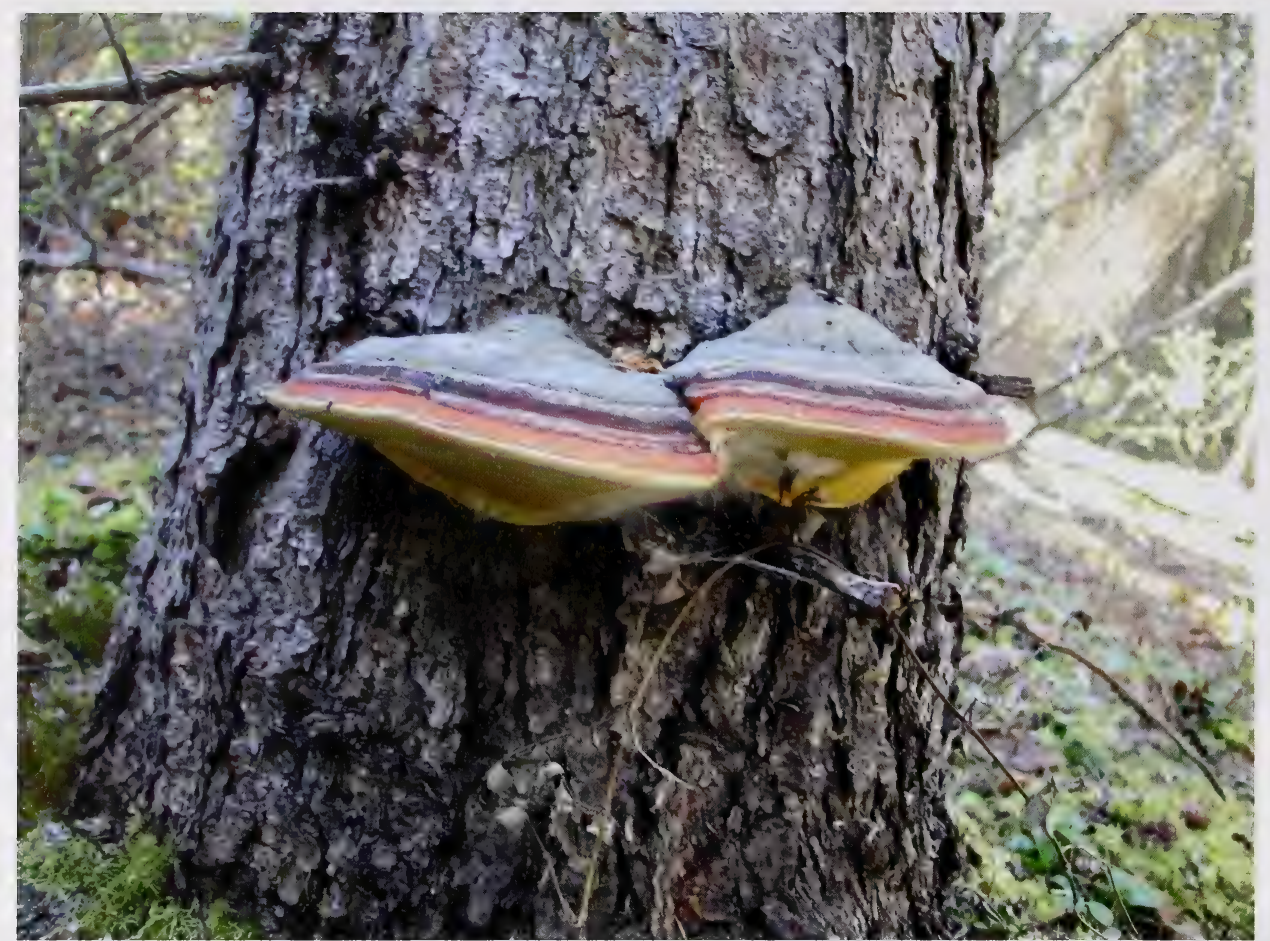

Fomitopsis pinicola On black spruce, Colin-Cornwall Lakes

Wildland Provincial Park

Photographer: Drajs Vujnovic

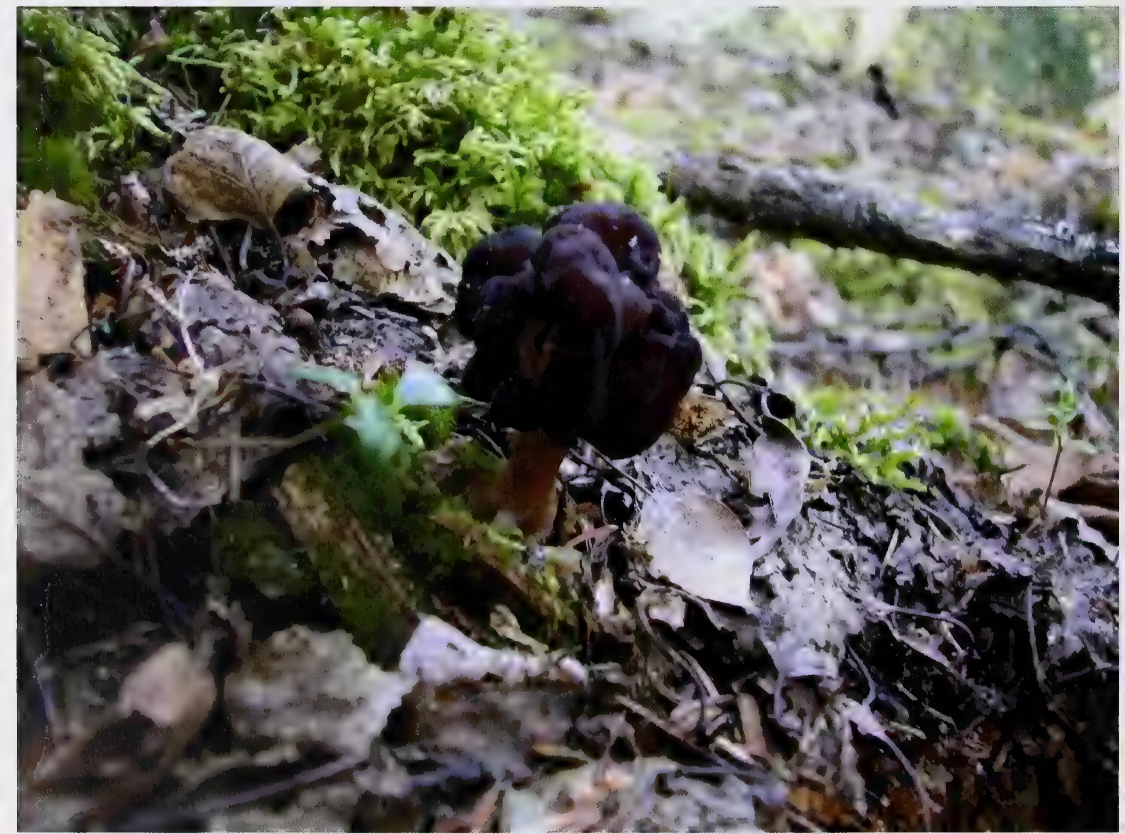

Gyromitra esculenta, Colin-Cornwall Lakes Wildland Provincial Park

Photographer: Drajs Vujnovic 

National Library of Canada
Bibliothèque nationale du Canada

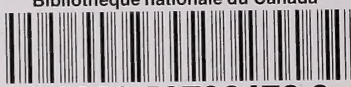

33286527364786 